\title{
Norois
}

Environnement, aménagement, société

$246 \mid 2018$

Eaux, marais, lac, étang, pêche

\section{Le patrimoine, un élement fédérateur pour la gouvernance des espaces protégés ? L'exemple du marais de Brouage (Charente-Maritime)}

Heritage: a unifying factor for the governance of protected areas? The example of the Brouage marshes (Charente-Maritime)

Mathilde de Cacqueray, Céline Barthon, Céline Chadenas, Xavier Michel, Vincent Andreu-Boussut et Arnaud de Lajartre

\section{OpenEdition}

Journals

Édition électronique

URL : https://journals.openedition.org/norois/6280

DOI : $10.4000 /$ norois. 6280

ISBN : 978-2-7535-7539-4

ISSN : 1760-8546

Éditeur

Presses universitaires de Rennes

Édition imprimée

Date de publication : 6 juin 2018

Pagination : 7-27

ISBN : 978-2-7535-7537-0

ISSN : 0029-182X

Référence électronique

Mathilde de Cacqueray, Céline Barthon, Céline Chadenas, Xavier Michel, Vincent Andreu-Boussut et Arnaud de Lajartre, « Le patrimoine, un élement fédérateur pour la gouvernance des espaces protégés ? L'exemple du marais de Brouage (Charente-Maritime) », Norois [En ligne], 246 | 2018, mis en ligne le 20 juin 2020, consulté le 14 janvier 2022. URL : http://journals.openedition.org/norois/6280 ; DOI : https://doi.org/10.4000/norois.6280 


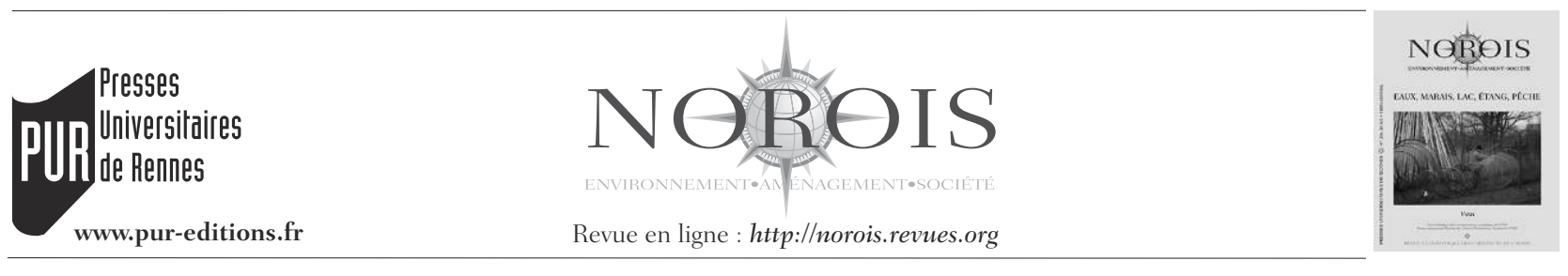

\title{
Le patrimoine, un élement fédérateur pour la gouvernance des espaces protégés? L'exemple du marais de Brouage (Charente-Maritime)
}

\author{
Heritage: A Unifying Factor for the Governance of Protected Areas? \\ The Example of the Brouage Marshes (Charente-Maritime)
}

\author{
Mathilde de Cacqueray ${ }^{a}$, Céline Barthon ${ }^{a *}$, Céline Chadenas ${ }^{b}$, Xavier Michel ${ }^{c}$, \\ Vincent Andreu-Boussut ${ }^{d}$ et Arnaud de Lajartre ${ }^{e}$
}

\footnotetext{
* auteur correspondant

a UMR 6590 Espaces et Sociétés, université d'Angers, Maison des Sciences Humaines - 5 bis boulevard Lavoisier,

49045 ANGERs.(mdc.univ.angers@gmail.com)(celine.barthon@univ-angers.fr)

b Géolittomer-Nantes UMR 6554 LETG, Campus du Tertre, BP 81227, 44312 NaNTEs cedex 3. (celine.chadenas@ univ-nantes. $f$ r)

c UMR 6590 Espaces et Sociétés, Normandie Université, Maison de la Recherche en Sciences Humaines, -

Esplanade de la Paix, 14032 CAEn cedex. (xavier.michel@unicaen.fr)

d UMR 6590 Espaces et Sociétés, université du Maine - avenue Olivier-Messiaen, 72085 LE Mans Cedex 9. (vincent.andreu-boussut@univ-lemans.fr)

e EA 4337 Centre J.-Bodin de recherche juridique et politique, faculté de droit - 13 allée F.-Mitterrand, 49036

ANGERS cedex 01. (arnaud.delajartre@univ-angers.fr)
}

Résumé : Partant du constat du caractère conflictuel de la protection écologique de l'espace à l'échelle locale, cet article analyse ce processus comme un moment de la patrimonialisation de l'espace et montre comment des enjeux de pouvoirs, d'appropriation et de mise en valeur participent à sa requalification et contribuent à l'émergence d'une nouvelle gouvernance territoriale. Appliqué à un espace artificiel gagné sur la mer, le marais de Brouage, asséché au XIX siècle et requalifié en «zone humide » menacée et à préserver au $\mathrm{Xx}^{\mathrm{e}}$, cette contribution s'attache dans une première partie à identifier ce qui fait patrimoine pour les parties-prenantes (gestionnaires d'espaces protégés, acteurs des territoires, socioprofessionnels et habitants - permanant et temporaire - ) avant d'analyser, dans un second temps, dans quelles mesures les objets, les biens et les valeurs qui leur sont associées peuvent fédérer, malgré des divergences, autour d'enjeux et de projets partagés privilégiant une coévolution et coadaptation entre nature et société. La méthode mobilisée recourt d'une part, à l'analyse des dires d'acteurs formulés dans le cadre d'entretiens semi-directifs et à la cartographie de leurs représentations spatiales du patrimoine et, d'autre part, à l'enquête par questionnaires permettant de recueillir la perception des usagers - permanents et temporaires - de l'espace et du patrimoine.

\footnotetext{
Abstract: Noting the conflictual nature of the environmental protection of spaces at local level, this article offers an analysis of this process as a period in which heritage status is conferred upon spaces, and shows how questions of power, appropriation and promotion play a role in the redefinition of these spaces and contribute to a new form of territorial governance. Applied to an artificial space reclaimed from the sea (the Brouage marshes), drained in the $19^{\text {th }}$ century, redefined as endangered "wetlands", and protected in the $20^{\text {th }}$ century, this contribution first of all identifies what constitutes a heritage space for the stakeholders concerned, before going on to analyse the extent to which the objects, material goods and values associated with heritage can be a unifying factor, in spite of diverging views, with regard to shared issues and projects promoting co-evolution and co-adaptation between nature and society. The method brought into play calls upon the analysis of opinions expressed by different actors in the course of semi-structured interviews, the mapping of their spatial representations of heritage areas, and a questionnaire-based survey revealing the perceptions of users - both permanent and temporary regarding the spaces and heritage areas concerned.
} 
Mots clés : patrimonialisation - perception - représentation - conflit d'usage - zone humide - gouvernance.

Keywords: heritage status - perceptions - representations - conflicts of use - wetlands - governance.

\section{INTRODUCTION}

La création d'espaces protégés au titre des sites, paysages, habitats, espèces, réseaux et continuités écologiques (Lepart et Marty, 2005) est rarement consensuelle car elle projette sur l'espace de nouvelles normes et valeurs qui tendent d'une part à modifier les rapports au lieu et à la nature du lieu et, d'autre part, à instaurer des nouvelles règles et modes d'habiter (Robert et Melin, 2016; de Lajartre et al., 2016). Ainsi, la protection de la nature hier et celle de la biodiversité aujourd'hui créent-elles une nouvelle catégorie d'espace ${ }^{1}$ - et en requalifient d'autres - en s'appuyant sur une expertise écologique souvent déconnectée de la "réalité » sociale des milieux (définis comme naturels) et sous-estimant l'importance des valeurs culturelles de certains d'entre eux.

Dès lors, la question de l'acceptation sociale de la protection et l'accompagnement aux changements produits par celle-ci deviennent un véritable leitmotiv pour les pouvoirs publics comme pour les gestionnaires avec, pour enjeu-cadre, l'articulation entre développement durable du territoire et conservation de la biodiversité. L'espace protégé devient ainsi un espace de transactions multi-scalaires entre différentes partie-prenantes dont les attitudes et comportements de rejet ou de consentement face à la protection peuvent évoluer au fil du temps, des contextes et des projets. Des travaux récents (dont Depraz, 2015; Laslaz, 2015; Claeys, 2000, 2014; Cadoret, 2011 ; Torre et al., 2010) ont ainsi démontré l'importance d'analyser les conflits et leur dynamique comme « une opportunité de mise en relations des acteurs », " un mode d'apprentissage du lien social »(Depraz, 2015), ou encore « une construction sociale de la relation à l'espace protégé » (Laslaz, 2015). Ils peuvent également être lus comme un moment de la patrimonialisation de l'espace mêlant des enjeux de pouvoirs, d'appropriation de l'espace et de légitimation (Veschambre et

1. Les espaces naturels protégés (ENP) dont la géographie a été analysée par Depraz, 2008.
Ripoll, 2005), tant pour l'identification et la sélection de ce qu'il faut protéger contre les effets du temps ou du risque de disparition, que pour ce qui relève de la transmission et de la valorisation (Gravari-Barbas, 2005; Veschambre; 2007, 2008; Bouisset et Degrémont, 2013 a et b). D'ailleurs, si la fabrique du patrimoine est potentiellement conflictuelle quels que soient les objets, les lieux et espaces concernés, elle est aussi porteuse de sens et de projets car :

- elle traduit « un fait du présent qui opère un tri sur le passé » (Péron, 2002) sur ce qui est - ou perçu - comme menacé afin d'en assurer la transmission ;

- elle fédère et mobilise des individus et des groupes sociaux qui se reconnaissent à partir d'un ensemble d'objets, de biens et de valeurs considérés comme dignes d'être préservés pour les générations futures;

- elle génère de nouvelles formes de mobilisations entre acteurs aux identités multiples et aux ressources asymétriques autour de biens et d'enjeux communs, en inventant de nouvelles organisations, coopérations et ententes contribuant à la gouvernance des territoires (Rey-Valette et al., 2014).

Notre article s'inscrit dans ce champ de la recherche sur le patrimoine et pose l'hypothèse que la patrimonialisation de la nature est à la fois porteuse de conflits tout comme des conditions d'acceptation et d'adaptation aux changements produits. D'où l'intérêt de mettre en perspective «ce qui fait patrimoine » pour les habitants et acteurs mobilisés dans la gestion de l'espace en contrepoint des zonages de protection issus de l'expertise écologique afin d'identifier, d'une part, la diversité des biens et des objets considérés comme signifiants, leurs valeurs et les enjeux associés; et, d'autre part, d'en déduire les potentiels tensions et conflits mais également les coopérations susceptibles d'émerger autour de projets favorisant l'intégration de l'espace social et de la nature pour un partage plus équitable de l'espace protégé. Ainsi, le processus de patrimonialisation de la nature dépasserait les logiques de 
rupture et de résistance «face à l'irréversibilité affichée de la détérioration de la nature » (Hériter et Guichard, 2008) pour s'inscrire dans une dynamique de résilience «empreinte de coévolution, de coadaptation, d'intimes associations entre nature et société et faisant appel à une modernité renouvelée où prime la réflexivité » (Berdoulay et Soubeyran, 2013).

Le terrain investigué ici est le marais de Brouage ${ }^{2}$ (Charente-Maritime), un milieu artificiel, dont les paysages gagnés sur la mer et le golfe de Saintonge dès le Moyen Âge pour l'exploitation du sel en portent encore les traces malgré les travaux d'assainissement (fin XVIII ${ }^{\mathrm{e}}$-début $\mathrm{XIX}^{\mathrm{e}}$ siècle) pour y implanter l'élevage et la production de fourrage, puis de drainage (fin des années 1970-1980) pour la céréaliculture intensive (Réault-Mille, 2003). Identifié à « une ressource naturelle et spatiale pour des usages ou aménagements dépendants des sociétés urbaines »(ibid.), il est successivement passé d'un marais-salant dont la production était vouée à l'exportation, à un marais « réserve foncière » dédié à l'élevage et aujourd'hui plébiscité pour ses paysages palimpsestes et sa biodiversité.

Son entrée en politique environnementale (Kalaora, 2010) et sa dynamique de patrimonialisation sont d'ailleurs contemporaines au déclin de l'élevage et à la déprise du marais (1970-1980) et reposent sur la création successive - non sans heurt - de la Réserve naturelle nationale de Moëze-Oléron en $1985^{3}$, d'un périmètre Natura 2000 en 2004 et $2009^{4}$ et du classement au titre des sites en $2011^{5}$ en raison de «sa singularité et de ses composantes pittoresques et historiques, celles-ci étant, avec l'argument scientifique, les deux principales catégories d'intérêt identifiées et mises en exergue pour classer ce site » (Ollier et Provost, 2009) (figure 1 - planche I et figure 2). Notons enfin, la création du $7^{\text {e }}$ Parc naturel marin de France sur l'estuaire

\footnotetext{
2. Un terrain parmi les quatre du programme «Gouvernance et développement durable des espaces du patrimoine naturel littoral » (baie de Somme, Parc naturel régional de la Narbonnaise en Méditerranée, marais de Brouage et Grand-Cul-de-Sac-Marin en Guadeloupe) financé par le Fondation de France (2013-2017), qui privilégie des zones humides littorales considérant ces espaces comme révélateurs des enjeux de protection de la biodiversité tout en faisant l'objet d'usages encore diversifiés et souvent conflictuels.

3. [https://www.legifrance.gouv.fr/jo_pdf.do? cidTexte=JORFT EXT000000689004].

4. [https://inpn.mnhn.fr/site/natura2000/FR5400431].

5. [http://www.poitou-charentes.developpement-durable.gouv.fr/brouage17-site-classe-r885.html].
}

de la Gironde et la mer des Pertuis en $2015^{6}$ dont le périmètre marin intègre l'interface terre-mer des marais charentais alimentés par la Charente dont la gestion intégrée de l'eau, en quantité comme en qualité, représente la clef de voute de la gouvernance environnementale à l'échelle du bassin et de l'avant-pays côtier (EPTB Charente, 2014).

\section{MÉTHOdologie}

L'article repose sur le traitement de données issues d'enquêtes de terrain réalisées sur le marais de Brouage entre 2014 et 2016. Dix-neuf entretiens semi-directifs enregistrés et/ou filmés auprès de différents acteurs de ce territoire (tableau 1) ont été retranscrits et analysés manuellement en fonction d'une grille organisée en quatre grands thèmes structurants (gouvernance et projet; gestion des espaces naturels et des usages associés; identification et valeurs du patrimoine; évaluation du changement, prospective et innovation) et sous-thèmes (ex. pour le patrimoine : objets et biens identifiés, lieux et espaces, valeurs, menaces) permettant de positionner les discours de chaque interviewé et d'identifier leurs principales récurrences, oppositions ou divergences. Parallèlement, l'analyse verticale des entretiens nous a permis de produire une synthèse individuelle par acteur et catégorie d'acteurs mettant en avant leurs perceptions de l'espace (à différentes échelles géographiques, protégé ou non...), du patrimoine, des acteurs et des enjeux associés à la gestion du marais.

Notons également qu'une carte du marais et de ses abords ${ }^{7}$ leur était soumise pendant l'entretien afin que chaque acteur y localise et développe ce qui faisait patrimoine pour lui.

Enfin, 200 questionnaires auprès des habitants permanents et temporaires (touristes) ont été réalisés afin de connaître - entre autres - leur perception du marais et surtout, leur vision du patrimoine à conserver $^{8}$.

\footnotetext{
6. [https://www.legifrance.gouv.fr/eli/decret/2015/4/15/DEVL1133922D/jo]. 7. Carte IGN format A4 noir et blanc (Géoportail), allant de l'estuaire de la Charente à celui de la Seudre et intégrant les 11 communes du marais de Brouage (Hiers-Brouage, Marennes, Bourcefranc, Saint-Just-Luzac, Saint-Froult, Saint-Sornin, Saint-Agnant, Saint-Jean-d'Angles, Moëze, Beaugeay, La Gripperie-Saint-Symphorien).

8. Les questionnaires ont été passés sur le terrain par Floriane Marchais, étudiante en Master 2 « Chef de projet, conseil en développement durable du tourisme » de l'université d'Angers, en été 2014. Composés de 6 volets : 1) cadrage des pratiques spatiales;2) pratiques des sites;3) images et sentiments développés pour les sites; 4) connaissance et appréciation de
} 


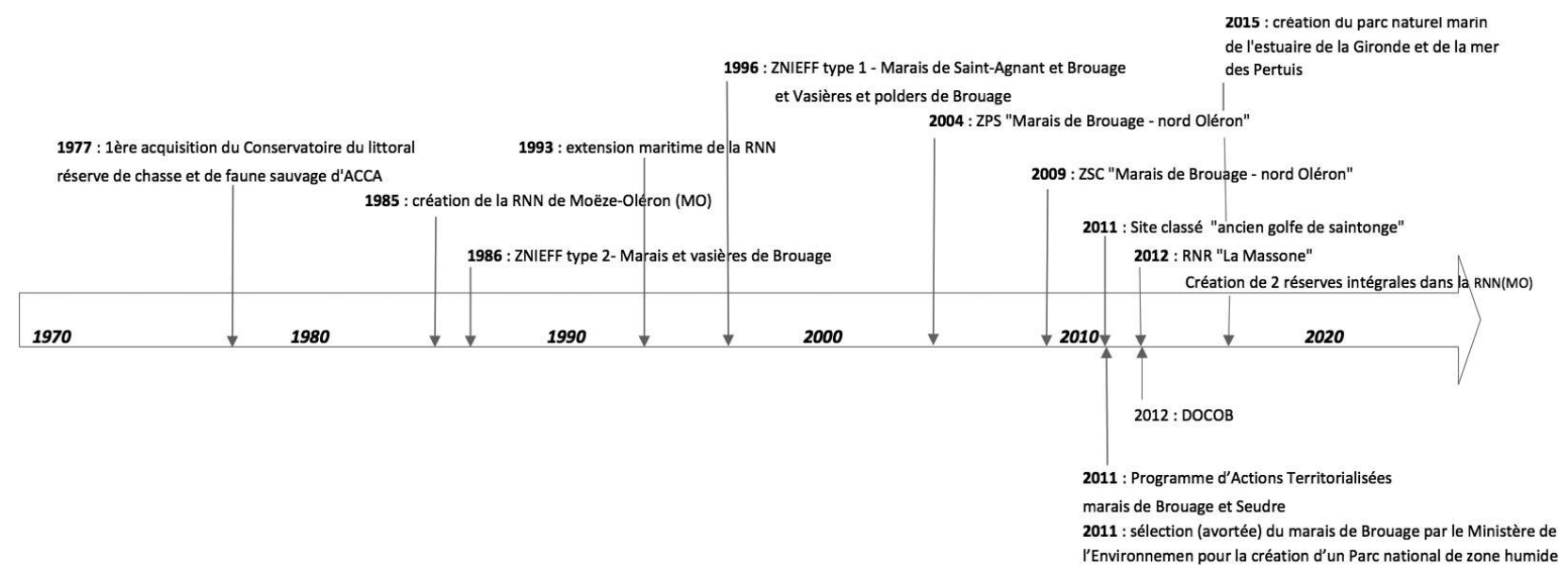

Figure 2 : Frise chronologique de la protection de la nature et des sites dans le marais de Brouage (Source : INPN. Réalisation Céline Barthon - UMR-ESO Angers)

Légende : ACCA : Association communale de chasse agréée; RNN : Réserve naturelle nationale; ZNIEFF : Zone naturelle d'intérêt écologique floristique et faunistique; ZPS : Zone de protection spéciale (Natura 2000); ZSC : Zone spéciale de conservation (id.); RNR : Réserve naturelle régionale.

Timeline of nature protection and site protection in the Brouage marshes

\begin{tabular}{|l|l|}
\hline \multicolumn{1}{|c|}{ Catégories d'acteurs } & \multicolumn{1}{c|}{ Structures et/ou acteurs } \\
\hline A) Services de l'État & (1) DREAL - inspection des sites \\
\hline B) Établissement public d'état & (2) Conservatoire du Littoral \\
\hline C) Collectivités territoriales & $\begin{array}{l}\text { (3) (4) (5) (6) Élus communaux } \\
\text { (7) (8) Chargés de mission de la communauté de commune de Marennes } \\
\text { (Natura 2000, gestion intégrée de la zone humide) }\end{array}$ \\
\hline $\begin{array}{l}\text { D) Gestionnaires, techniciens d'espaces natu- } \\
\text { rels, animateur nature }\end{array}$ & $\begin{array}{l}\text { (9) (10) Réserve naturelle nationale (RNN) de Moëze-Oléron } \\
\text { (11) Pôle nature 17 (Conseil départemental) } \\
\text { (12) (13) Forum des marais Atlantique }\end{array}$ \\
\hline E) Association naturaliste & (14) (9) (10) Ligue de protection des oiseaux (LPO) \\
\hline F) Association de chasseurs & (15) (16) Fédération départementale de la chasse de Charente-Maritime \\
\hline G) Professionnels de l'agriculture & $\begin{array}{l}\text { (3) Céréalier } \\
\text { (17) (18) Éleveur }\end{array}$ \\
\hline H) Associations syndicales de marais & (3) (17) \\
\hline I) Organisme de développement touristique & (19) Office de tourisme du bassin de Marennes Oléron \\
\hline
\end{tabular}

Tableau 1 : Catégories d'acteurs rencontrés dans le cadre des entretiens

NB : Certains acteurs font partie de plusieurs catégories. Les numéros indiqués servent de références aux citations dans le texte. Categories of actors and stakeholders interviewed

C'est donc le croisement de ces enquêtes qualitative et quantitative qui nous a permis d'apporter des éléments de réponses aux questionnements suivants :

la protection; 5) vision et représentation de ce qui « fait patrimoine »; 6) profils sociodémographiques, leur passation devait respecter un quota entre population résidente à l'année (Insee, 2011) et population temporaire, appréhendée à partir de la capacité d'accueil touristique des 11 communes du marais (Insee, 2014). À noter que seuls les volets 3 et 5 seront mobilisés ici. Leur traitement sur le logiciel Sphinx repose sur une analyse statistique et textuelle des données.
- Quels sont les biens ou les objets (matériels et immatériels) faisant patrimoine, c'est-à-dire considérés comme un héritage commun, dignes d'être transmis aux générations futures et par conséquent à conserver ?

- Observe-t-on des convergences ou des divergences d'appréciation et de reconnaissance du patrimoine entre ces différents groupes d'acteurs?

- Comment intégrer ces différentes représentations du patrimoine dans la gestion et la valorisation 
de l'espace protégé? Et pour quelle gouvernance partagée du territoire?

\section{DÉlimitation SPATIALE ET REPRÉSENTATION DU PATRIMOINE POUR LES ACTEURS ET HABITANTS DU MARAIS DE BROUAGE}

\section{Un « tout $» . .$.}

À la question «qu'est-ce qui fait patrimoine pour vous », la population enquêtée par questionnaire (figure 3 - planche II) et les différents acteurs interrogés lors des entretiens développent, dans un premier temps, une vision globale qui se recoupe en de nombreux points.

Si la citadelle de Brouage et son patrimoine historique, bâti et architectural sont généralement cités en premier lieu quelles que soient les catégories d'acteurs et la population enquêtée, le marais " dans son ensemble » apparaît ensuite comme une évidence, un « tout » non dissociable ni réductible à une somme d'objets ou de sites particuliers "Brouage bien sûr ça c'est clair, on va commencer par ça [...] mais moi, je vais tout vous cocher, je vais vous cocher l'ensemble du marais [...] tout ce marais à bosses " (4); " bien sûr tout [le marais] est à conserver. Il faut tout conserver en conservant les acteurs [...]. Il faut absolument conserver nos activités qui justement permettent d'avoir ce marais » (14). "Le marais dans sa totalité. » (3). "Le marais tel qu'il était avant et surtout qu'il reste ce qu'il est [...]. Parce que ça c'est un patrimoine à lui tout seul » (18).

Cette première lecture est d'ailleurs visible sur la carte de synthèse des représentations spatiales du patrimoine des acteurs interrogés (figure 4 planche II) où figurent des périmètres imbriqués entre eux, allant du cœur du marais (nuance de vert foncé) aux marges de celui-ci (vert clair), plus que des lieux en particulier.

Notons que ces zonages intuitifs et empiriques se superposent en partie aux zonages de protection institutionnels (figure 1 - planche I) avec néanmoins une surreprésentation du marais « à bosses » anciennement salant au centre et dans la partie interne du marais (nuance de vert foncé), par opposition aux marais plats, doux en lisière des coteaux de l'ancien golfe de Saintonge ou asséchés, issus de la poldérisation et du drainage intensif à l'ouest de Brouage et sur la commune de Moëze. En revanche, la partie maritime apparaît en retrait, malgré la présence de l'ostréiculture et de la réserve naturelle, symbole de la rupture entre la mer et le marais depuis les travaux d'assainissement du préfet Le Terme au début du XIX siècle transformant le marais salicole en déprise, en marais d'élevage désormais alimenté de manière indirecte par le canal de la Seudre à la Charente et celui de Brouage.

D'ailleurs un élu d'une commune située en fond de marais nous confie que si «l'ensemble du marais fait patrimoine [lui] n'irait même pas jusqu'à Moëze parce qu'après vous avez le marais maritime. Du côté de Moëze, alors là c'est plus LE marais quand même parce que vous avez de l'eau salée; ici vous n'avez que de l'eau douce [...]. Ce sont deux particularités quand même... entre un marais salé ce n'est pas la même faune que le marais doux. Ce n'est pas du tout comparable» (5). Rares sont aussi les habitants enquêtés par questionnaires à avoir caractérisé le patrimoine du marais de « littoral » (5 citations) ou « côtier» (3 citations), préférant qualifier l'espace de «naturel » ou de « nature » sans toutefois citer ces deux items pour qualifier le patrimoine (4 citations).

Une lecture plus fine des dires d'acteurs permet également de saisir l'importance de l'eau pour la totalité d'entre eux, mais souvent de manière indirecte, en lien avec l'histoire et les usages, insistant tantôt sur les aspects matériels (eau ressource structurant le paysage, les aménagements et les écosystèmes) ou immatériels (pratiques, imaginaire et savoir-faire) : "Pour moi c'est très clair, [le patrimoine] c'est l'histoire du marais, ce sont les oiseaux, ce sont les agriculteurs, c'est la biodiversité et c'est surtout l'eau [...]. Il y a un règlement de l'eau qui a été fait par Le Terme en 1826 et donc le plan de gestion du marais, il existe depuis deux siècles et il se transmet de génération en génération et de père en fils. Et c'est parce qu'il y a ça qu'il y a des loutres aujourd'hui, qu'il $y$ a des cistudes, qu'il y a des oiseaux, sinon il n'y aurait pas!» (2). «Pour moi c'est le réseau hydraulique; ça structure le paysage " (7). "Moi je vais dire qu'il y a ce réseau de fossés, toute la gestion de l'eau dépend de ça [...]. Il y a quand même un patrimoine aussi autour de l'eau, tout ce que j'appellerai le savoir-faire, c'est du patrimoine culturel $\gg(15)$.

L'eau, son contrôle, sa gestion et tout le savoir-faire qui y est associé révèlent également l'importance accordée aux hommes et à leurs activités sans les- 
quels, le marais n'existerait pas. «Ce sont les hommes qui font le marais » (16). Le patrimoine du marais de Brouage «c'est l'activité (élevage) et c'est sa biodiversité » (8). "Moi ce que je vois à l'intérieur de cette zone-là, c'est tout ce qui est milieu on va dire plus ou moins naturel; après des parties plus fonctionnelles, le réseau hydraulique, la voirie, tout ce qui est activités, les savoir-faire liés à ces activités-là, le patrimoine immobilier (la citadelle, les tonnes de chasse). Et estce qu'il faut qu'on mette du coup qu'on conserve des hommes dans le marais?» (15). Pour la majorité des acteurs - céréaliers exclus -, l'élevage extensif reste l'activité majeure permettant d'entretenir le milieu et ce, depuis le XIX ${ }^{\mathrm{e}}$ siècle, où les premières associations syndicales de marais ont été créées. Le déclin de celui-ci est d'ailleurs perçu comme la principale menace de disparition, face aux logiques de drainage des années 1980 et au manque de renouvellement des exploitations, bien plus que le changement climatique et le risque de submersion, rarement cités de manière spontanée par les acteurs.

«La menace, c'était que le marais de Brouage soit aplani, soit transformé, drainé, avec Saint-Laurentde-la-Prée, à côté, qui avait mis en place les schémas de drainage qui fonctionnaient bien ${ }^{9}$. On perdait toute cette valeur paysagère, historique [...]. Donc on s'est mis à acheter et ça a été compliqué parce que, à l'époque [...], ce n'était pas la mode au Conservatoire de s'occuper de marais, parce que le drainage n'était pas inventorié comme étant une menace comme la construction et l'urbanisation. » (2)

On retrouve d'ailleurs l'expression de cette menace pour les habitants enquêtés pour qui « l'agriculture intensive », « les intrants », « la culture du maïs » et "l'assèchement du marais » figurent en tête des réponses relatives à la perception des risques pesant sur le marais, bien avant « la chasse », « l'homme », «le tourisme» ou «le changement climatique» (termes cités par les enquêtés).

Les marais asséchés endigués utilisés pour la céréaliculture intensive sont par ailleurs exclus des périmètres patrimoniaux des naturalistes et des chasseurs (mais inclus dans ceux des agriculteurs

9. L’unité expérimentale de Saint-Laurent-de-la Prée a été mise à disposition de l'Inra en 1964 pour développer l'agriculture dans le marais grâce à un réseau hydraulique drainant l'eau du sol. L'objectif est alors d'intensifier les systèmes culturaux en privilégiant les grandes cultures et en rationnalisant le réseau hydraulique. Les axes prioritaires d'expérimentation sont alors l'hydraulique, l'agronomie et l'élevage.
- céréaliers - dont c'est le métier) tout comme du périmètre Natura 2000 dans lequel la majorité des habitats et espèces identifiés dépend de l'élevage et de la gestion de l'eau.

"Une perte de biodiversité telle qu'on l'a identifiée et recensée actuellement, pour moi, ce serait si on n'avait plus assez d'élevage. Si on n'a plus d'élevage en fait, on va perdre pas mal de pratiques, on va perdre l'hétérogénéité du milieu, donc des habitats et les espèces qui y sont associés. » (7)

Composite, hybride, entre culture et nature, le marais-patrimoine ne saurait donc se résumer à des habitats et des espèces à protéger sans prendre en compte les héritages passés et sans se questionner sur le projet écologique et social à y développer. Et si la notion de paysage culturel évolutif peut nous paraître ici la plus appropriée pour caractériser le patrimoine à transmettre, les valeurs qui lui sont attribuées se superposent ou diffèrent d'un groupe à l'autre.

\section{Des valeurs...}

Transformer un objet - naturel ou culturel - en bien commun, convertir un lieu, un site ou un espace en patrimoine digne d'être préservé pour les générations futures conduit indéniablement à lui affecter une nouvelle valeur (Barthon et al., 2013). Matérielle ou idéelle son évaluation reste cependant difficile à analyser d'une part en raison de la polysémie de la notion de valeur « comme-mesure, comme-préférence ou comme-norme » et, d'autre part, du fait de la forte intrication de celles-ci (Maris et al., 2016). Pour Bouisset et Dégremont, 2013-c, "l'imbrication des valeurs dépasse les distinctions entre patrimoine naturel et patrimoine culturel, l'appréciation des deux va de pair ». Ainsi les valeurs économique, fonctionnelle, écologique mais aussi esthétique, culturelle, symboliques, affective ou de legs se retrouvent-elles mobilisées dans tout processus de patrimonialisation, bien qu'inégalement reconnues et partagées entre catégories d'acteurs.

Celles associées au marais-patrimoine sont donc multiples et peuvent, à l'image des travaux réalisés par Maris et al. (2016) se regrouper en 3 catégories, écologique, instrumentale et culturelle, pour lesquelles les appréciations des acteurs interviewés se recoupent ou s'opposent. Indéniablement, c'est la 
valeur culturelle qui est la plus partagée, d'abord à travers l'histoire dont les marqueurs/repères se traduisent par la citation des monuments historiques et de l'évolution du marais-paysage sur le temps long, mais aussi à travers l'expérience que les acteurs en ont. Ainsi, l'attachement au marais est manifeste pour les habitants-usagers, éleveur, chasseur, ostréiculteur, agriculteur (y compris intensif) pour qui le marais représente un espace de vie, de pratiques professionnelles et de loisir totalement imbriquées :

"Quand vous avez des gens en plus comme moi [chasseur et] aussi ostréiculteur c'est plus que tout... tout tourne autour du marais. » (15)

«Moi, le marais m'a fondé. Donc voilà je lui dois d'être ce que je suis. Et puis j'ai beaucoup d'affection pour lui quoi [...]. Le marais ce qui le caractérise c'est ce mélange de terre et eau qui fait qu'il y a une sorte de magie qui s'en dégage mais c'est difficile à traduire... c'est un sentiment plus qu'autre chose. » (17)

Les références au passé familial et à la transmission des savoir-faire sont fréquentes dans les témoignages où l'attachement au marais et le sens du lieu (Sébastien, 2016) se réfèrent plus à une histoire personnelle ou rattachée à un groupe social - indépendamment de leur hétérogénéité, les chasseurs comme les éleveurs ne formant pas un groupe homogène - qu'à "l'esprit des lieux » (1) tel que revendiqué par les services de l'État dans le classement du site.

D'ailleurs, si la valeur écologique est avant tout partagée par les services de l'État et les gestionnaires d'espaces naturels $(1,2,7,9,10,14)$, elle n'est pas étrangère aux éleveurs et aux chasseurs. Mais alors que les premiers privilégient avant tout les espèces, les habitats et les paysages naturels, quitte à voir le marais «s'ensauvager »- sans pour autant se fermer -, les seconds envisagent plutôt un marais jardiné, avec des canaux bien curés, sans flaque d'eau :

«Les acteurs [éleveur et/ou propriétaire foncier] du marais n'ont pas forcément la même considération que les services de l'État quand ils ont classé le site. On a un patrimoine qui d'un côté a été figé alors qu'eux ont plus en tête un patrimoine dynamique. Par exemple, pour les associations de protection de l'environnement cela ne les dérange pas de voir des bords de fossés abîmés, des berges effondrées... Au contraire ça peut très intéressant pour les espèces. Le propriétaire lui, en termes de fonctionnalités, si la berge est effondrée l'eau va moins bien couler, les vaches peuvent s'échapper. Donc on a des conceptions vraiment très très différentes en termes de vision du paysage et donc du patrimoine. » (8)

La focalisation sur les espèces patrimoniales est d'ailleurs discutée dans ses fondements, y compris par les gestionnaires considérant que la valeur globale d'un écosystème ne repose pas uniquement sur l'emblématique mais aussi sur le commun et l'ordinaire : "Le patrimoine, c'est la cerise sur le gâteau [...] si je vous donne la cerise et si je mange le gâteau, vous sortez de table en ayant faim et moi j'ai mal au ventre! [...] C'est prétentieux de vouloir croire qu'on est capable d'évaluer les indicateurs patrimoniaux isolés du reste! L'écologie c'est des êtres interconnectés » (9).

Enfin, la valeur instrumentale, de par son caractère multiple, soulève des tensions en raison notamment de l'appréciation de la valeur d'usage et économique du marais. Si pour les gestionnaires et techniciens d'espaces naturels, c'est la dynamique de déprise agricole du marais qui contribue à sa valeur patrimoniale (ici la biodiversité), pour les agriculteurs comme pour les éleveurs, c'est au contraire l'activité productive, rattachée au foncier, qui en fait l'intérêt. Pour les céréaliers, le marais n'aurait d'ailleurs «plus aucune valeur parce que l'agriculteur ne peut plus en vivre. Il y a plus que la valeur que l'on puisse avoir en disant: on a des zones humides qui sont là, il faut les préserver... mais pour moi il y a plus de valeur» (3). Cette nouvelle lecture du marais en tant que zone humide dont les fonctionnalités et services rendus dépassent la notion de biens productifs privés au profit des communs en est pourtant qu'à ces prémices. Elle reste d'ailleurs en grande partie portée par un corps de techniciens et par le Conservatoire du littoral pour qui l'enjeu est avant tout de faire reconnaître les bénéfices rendus par le marais pour la régulation et la qualité des eaux, l'atténuation des risques naturels (inondation et submersion), le maintien d'une biodiversité emblématique et le développement d'activités économiques issues des ressources produites mais aussi la valeur sociale et touristique générée.

"Ce qui prime aujourd'hui dans le discours c'est l'intérêt général : la prise en compte des fonctions 
pour l'intérêt général et les services [...]. Alors c'est l'offre de diversité mais c'est aussi les autres services liés au stockage de l'eau et aux services rendus par rapport aux activités humaines... Bien entendu c'est anthropocentré les services! Voilà, il y a les grandes fonctions : ça c'est le cadre général et ça c'est rentré dans le discours de tout le monde, les élus locaux etc. Ce qui est un petit peu plus compliqué anjourd'hui c'est de bien cerner tous les services, ce qu'on peut en attendre, ce qu'on est en train de perdre et ce qu'il faudrait garder voire amplifier » (12)

"Il est vrai que cette économie-là [tourisme, pêche, chasse] risque de disparaitre ou de s'amoindrir si le caractère paysagé de qualité, la présence de biodiversité, d'oiseaux et tout cela venait à décliner parce que s'il n'y a plus l'entretien nécessaire de ces milieux... Là ce serait dommage. » (13)

Finalement, si les valeurs affectées au maraispatrimoine peuvent paraître contradictoires, elles se recoupent néanmoins dans les enjeux de conservation et de transmission de celui-ci, qui mobilisent, malgré des attitudes et des regards différents, l'ensemble des acteurs.

\section{Des enjeux...}

Replacés dans le système maraichin atlantique en crise (Corlay, 1986), face à une dynamique de déprise du marais accentuée par les dysfonctionnements territoriaux et organisationnels endogènes et exogènes autour de la ressource eau en CharenteMaritime (Bouba-Olga, Boutry et Rivaud, 2009), les enjeux identifiés par les différentes catégories d'acteurs lors des entretiens ne sont pas nouveaux (figure 5). Ce qui l'est, ce sont leurs interrelations et interdépendances dans un contexte marqué par un changement de paradigme et d'échelle faisant du marais de proximité (enjeu local) en crise, une zone humide ${ }^{10}$ menacée (enjeux national et international) et à préserver.

Ainsi, l'eau, le maintien et la maîtrise de sa circulation selon les saisons et les usages du marais reste bien sûr l'enjeu majeur pour l'ensemble des acteurs interrogés. Bien commun, sa gestion suppose de

10. Le terme « zone humide » apparaît pour la première fois dans les années 1960 comme objet de préoccupation des naturalistes, et plus précisément des ornithologues (ministère de l'Écologie, de l'Énergie, du Développement durable et de la Mer, 2009). répondre à l'interdépendance des usagers (RéaultMille, 2003). Cependant, son partage - et indirectement celui de l'espace - est hautement conflictuel et oppose d'un côté les céréaliers et de l'autre les éleveurs, ostréiculteurs, chasseurs et gestionnaires de la réserve naturelle de Moëze-Oléron dont les besoins en eau douce diffèrent également selon les saisons tout en coïncidant l'été pendant la saison sèche. De plus, si historiquement la compétence hydraulique appartenait intégralement au syndicat de marais et donc aux propriétaires, les nouveaux modèles agricoles productivistes des années 19701980, la complexification de la réglementation sur l'eau ${ }^{11}$ et la prise en compte des enjeux environnementaux ont conduit à remettre en question les compétences de cette organisation professionnelle au profit d'une délégation d'une partie de celles-ci vers les collectivités territoriales (Gilardeau, 2008).

L'entrée de la nature, des enjeux de conservation de la biodiversité, de la qualité de l'eau dans le marais soulève donc de nouvelles problématiques de gestion des milieux et de gouvernance qui viennent se surimposer à un mode d'exploitation traditionnel en crise où la nature apparaît comme "muette " (Billaud et al., 1996) dans les premières négociations des années 1990. Pourtant, et paradoxalement, c'est bien ce modèle extensif qui en fait aujourd'hui l'intérêt écologique et paysager. La « sédimentation patrimoniale » (Bouisset et Dégrémont, 2013b) qui s'opère (figures 1 et 2 ) traduit donc une co-construction entre nature et société qu'il s'agit de renouveler dans un contexte où les objectifs écologiques «parlent » désormais : "On peut avoir un objectif qui prend en compte le maintien de telle ou telle activité, mais aucun objectif ne dira: il faut maintenir telle activité. C'est clair, Natura 2000 [soit la quasitotalité du marais et de son avant-pays maritime], c'est avant tout un objectif écologique [...]. Mais à part l'élevage, je ne vois pas quelle autre activité pourrait assurer cette fonction écologique » (7). L'élevage extensif est d'ailleurs mobilisé par le Conservatoire du littoral et la Réserve naturelle de Moëze via des

11. En lien notamment avec la Directive Cadre sur l'Eau [http://www.eaufrance.fr/s-informer/comprendre/la-politique-publique-de-l-eau/la-directive-cadre-sur-l-eau], la loi sur l'eau et les milieux aquatiques traduisant dans le droit français cette directive [http://www.eaufrance.fr/s-informer/comprendre/la-politique-publique-de-l-eau/la-loi-sur-l-eau-et-lesmilieux] ; la loi sur le Développement des Territoires Ruraux (DTR) du 23 février 2005, codifiée à l'article L 21 1-1 du code de l'environnement et la GEMAPI, gestion des milieux aquatiques et prévention des inondations (loi du 27 janvier 2014 de modernisation de l'action publique). 
Figure 5 : Des enjeux de conservation et de valorisation du marais-patrimoine conditionnés par la gestion hydraulique des milieux (source : enquête acteurs, Gouvernapat; réalisation : Mathilde de Cacqueray [UMR ESO Angers])

Issues relating to the conservation and promotion of marshland heritage affected by water-management measures

conventions passées entre le Conservatoire régional d'espaces naturels et les éleveurs pour entretenir leurs acquisitions foncières et maintenir les milieux ouverts, propices aux espèces protégées. Quant à la valorisation du patrimoine par le tourisme, transversale à l'ensemble des enjeux et multi-acteurs, elle dépend étroitement de l'accessibilité de la zone et de son ouverture qui, en l'état, reste confinée à la place forte de Brouage : "Une enclave touristique dans un monde rural» (2).

Dans ce contexte, le patrimoine et ses représentations peuvent-ils fédérer autour de projets de territoire et faire émerger 1) une gouvernance partagée et appropriée aux enjeux identifiés? 2) une nouvelle approche privilégiant une coévolution et coadaptation entre nature protégée et société?

\section{UNE GOUVERNANCE PAR PROJETS EN RÉPONSE AUX ENJEUX DE GESTION D'UN MARAIS-PATRIMOINE EN CONSTRUCTION}

Face aux enjeux identifiés (figure 5), la gouvernance se structure progressivement autour de différents projets de territoire qui permettent de fédérer les acteurs autour d'objectifs communs. L'analyse des entretiens permet ainsi d'identifier des réseaux d'acteurs, les coopérations qui fonctionnent et les tensions qui persistent ou émergent en fonction de l'évolution du contexte territorial.

\section{La gouvernance autour du partage de l'eau}

L'enjeu qui mobilise l'ensemble des acteurs et illustre la complexité des réseaux est incontesta-

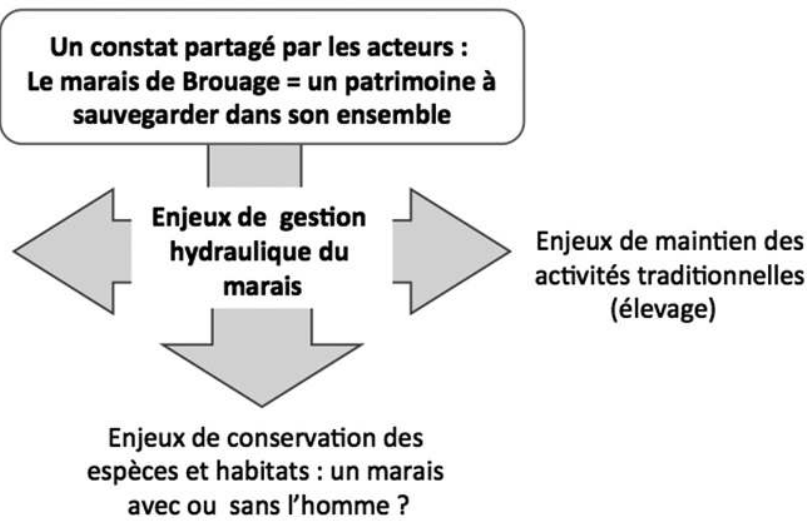

blement celui de la gestion hydraulique du marais. Les besoins en eau, en quantité comme en qualité, diffèrent considérablement d'un usage et d'une catégorie à l'autre (figure 6) : "Vous verrez très vite que l'eau est à la fois un lien entre les différents usages, les différents acteurs, mais aussi une source de contentieux» (8).

Si les conflits entre agriculteurs/ostréiculteurs autour de l'accès et le partage de cette ressource dans les marais charentais ont fait l'objet de nombreux travaux (Candau, Deldrève et Deuffic, 2012; Bossuet et Boutry, 2012; Rivaud, 2009; Bouba-Olga, Boutry et Rivaud, 2009; Bry et Hoflack, 2004), soulignons que la profession agricole reste divisée entre d'une part, les céréaliers, présentés comme l'élite sociale du marais (Billaud, 1996) incarnant le modèle productiviste des années 1980 et dont les besoins en eau au printemps et en été sont conséquents et, d'autre part, les éleveurs pour qui la quantité et la qualité doivent être constante pour l'alimentation et le regroupement des troupeaux à partir du printemps. Par ailleurs, les chasseurs à la tonne ont également des besoins : « 300 tonnes de chasse et leurs mares à alimenter fin août... Donc c'est vraiment à la même période [l'été] que ça devient très conflictuel [...] L'eau c'est vraiment le nerf de la guerre » (8). La réserve naturelle a également ses exigences en termes de niveau d'eau afin de maintenir les habitats propices aux espèces qu'elle protège. Face à cela, de fortes tensions sont apparues entre les gestionnaires, les chasseurs à la tonne et les agriculteurs en été 2015 : «Nous [Réserve naturelle de Moëze] sur un cycle annuel complet pour nos 300 hectares, la quantité prélevée est de l'ordre de $340000 \mathrm{~m}^{3}$ les années où on utilise le plus d'eau. Chaque année, au premier remplissage des mares de 
tonnes en août, période généralement de sécheresse, c'est $1250000 \mathrm{~m}^{3}$ pour un remplissage. Nous ont a été soumis à une enquête d'utilité publique mais pas eux...» (9). Pour les irriguants "Le plus gros des problèmes c'est quand l'été vous refusez de l'eau aux irriguants pour irriguer le maïs alors que les gens de la LPO remplissent les marais avec des pompes tout l'été» (3).

Le partage de l'eau en période de sécheresse, fréquente en Charente-Maritime, reste donc hautement conflictuel. Du protocole d'aménagement et de gestion concertés des marais charentais de 1991, $1^{\text {er }}$ acte de la gouvernance multi-acteurs du marais et de l'interface terre-mer, aux autorisations spéciale ou dérogatoire de la préfecture pour le prélèvement en fonction des usages et des risques de pénurie, on s'achemine aujourd'hui vers un véritable règlement de l'eau sur le marais : "C'est on va dire [le produit d'] une dizaine d'années de fort contentieux. Ce sera un arrêté, car on voit bien que de manière contractuelle on ne peut pas y arriver; ce sont toujours les mêmes [les céréaliers] qui ouvrent les vannes; on n'y arrive pas. » (8). Le principe d'un volume à répartir entre les différents usages actuels a été posé avec une hiérarchisation définie suivant les principes de la directive cadre sur l'eau, lui procurant une assise réglementaire.

L'ordre des priorités ${ }^{12}$ ainsi que la durée de validité de ce règlement (de 5 à 10 ans) inaugurerait donc un véritable tournant dans la gestion du marais dont la place est désormais reconnue par l'EPTB (établissement public territorial de bassin, 2014) et le SAGE (schéma d'aménagement et de gestion des eaux) de la Charente dans le système hydraulique du bassin. Il produirait également une petite révolution dans les associations syndicales de marais pour qui, traditionnellement « on ne peut gérer l'eau administrativement [...] l'eau ne se gérant bien que près » (Réault-Mille, 2003) et dont l'indépendance est désormais remise en question : déjà «aujourd'hui il y a plus de marge de manouvre parce qu'on vous dit, voilà vous avez un calendrier à l'année qui vous dit voilà vos jours d'ouverture et vos jours de fermeture. Au début, c'était nous, association syndicale de marais avec des associations syndicales d'ostréiculture. Maintenant c'est l'État. Avant il y avait deux réunions

12. 1. Alimentation en eau potable, 2. Maintien des niveaux d'eau dans la zone humide, 3. Irrigation, 4. Autres usages (remplissage de mares de tonnes de chasse notamment). par an, entre syndicats agricole et ostréicole. Et même si parfois on avait des choses à se dire [sous-entendu conflictuelles] au contraire on pouvait se les dire, alors que maintenant on peut plus se les dire parce qu'on se voit plus. » (3).

Les techniciens du Forum des marais atlantiques confortent cet adage de « la bonne gestion de proximité » et soulèvent parallèlement un autre problème en lien direct avec la transmission orale des connaissances techniques et des savoir-faire : "Il y a une vraie inquiétude sur le renouvellement des gestionnaires des associations syndicales dans la décennie qui vient puisqu'il y a assez peu de repreneurs... Donc l'État après va hériter du devoir de gestion en cas de vacance de la gestion. Comment va-t-il faire? Est-ce qu'il va pouvoir mettre des fonctionnaires? Est-ce qu'il va déléguer aux collectivités locales? [...] Un gestionnaire de marais sait très bien que quand il va tomber 5 millimètres, un petit orage sur tel secteur $d u$ marais, que dans les 3 heures qui suivent ça va monter de $5 \mathrm{~cm}$, il va le mesurer sur le caillou. On sait qu'il y a une marque là-bas sur le caillou à tel endroit. Si c'est monté très rapidement depuis la veille il va falloir qu'il aille ouvrir tel et tel ouvrage de deux dents ici, trois dents là-bas. Or, ces choses-là, c'est écrit nulle part! C'est dans la tête vraiment des éclusiers, quand il y en a un, et pour transmettre cela il faut des années en général. Quand il y a aura cette vacance du savoir-faire, il y aura un vrai problème, même si une collectivité locale doit prendre en main la gestion hydraulique $»(12)$.

Enfin, il faut prendre en compte le changement d'objectifs qu'ont connu ces associations syndicale de marais initialement créées pour l'assainissement et l'assèchement du marais et aujourd'hui orientées vers la conservation de la zone humide: «Les associations syndicales ont été constituées au départ, leur objet initial c'était l'assèchement, l'assainissement $d u$ marais. Autrement-dit, faire en sorte qu'il y ait le moins d'eau possible et que ça devienne des terres cultivables. Et puis il y a eu un infléchissement, une modification des priorités dans les années 2000 qui a débouché sur l'ordonnance du premier juillet 2004 qui a revu l'objet des associations syndicales qui maintenant sont au contraire chargées de contribuer à la préservation des zones humides. Ce n'est pas du tout la même chose mais l'objet reste un objet eau. » (17). Si l'objet «eau » reste le même, le patrimoine à transmettre en lien avec cette ressource change 
cependant d'enjeux et de fonction, invitant à repenser de nouvelles formes de coévolution/coadaptation nature-société au sein du marais.

D'ailleurs, si les représentations du marais-patrimoine varient en fonction des acteurs, tous s'accordent sur l'idée qu'il s'agit désormais de le «sauver» : «Tout le monde dit "non faudrait pas que ça disparaisse". [...] Alors ce qui se passe, la chance que l'on a, c'est qu'effectivement tout le monde tire dans le même sens [...] Ils ont compris que c'est tous ensemble... sinon on est mort » (18). Les acteurs semblent donc avoir pris conscience des menaces (endogène et exogène) qui pèsent sur le marais et de l'importance d'agir collectivement, en s'appuyant sur des dispositifs existants : "Ils se rendent compte, et c'est pour ça que les projets ont autant d'écoute, que le marais est en train de péricliter et qu'il faut faire des choses rapidement et de manière importante car si elles ne sont pas faites dans les cinq années qui viennent probablement on ne pourra plus rien faire » (8).

Une nouvelle gouvernance se structure donc autour de la restauration et de l'entretien du réseau hydraulique qui apparaît fondamental quels que soient les usages de l'eau. C'est donc autour d'un projet de contrat de territoire milieu aquatique (CTMA $)^{13}$ que se fédèrent aujourd'hui les acteurs. Porté par les intercommunalités de Rochefort et de Marennes, ce contrat est financé en grande partie par l'agence de l'eau Adour-Garonne et le soutien des conseils régional et départemental. Le Forum des marais Atlantique est là en soutien technique et en relais entre l'Agence de l'eau et les intercommunalités. Plusieurs actions sont d'ailleurs programmées dans le cadre de ce contrat dont les objectifs croisent enjeux hydrauliques, biodiversité et élevage, légitimant de fait « la zone humide » et les catégories d'acteurs s'y référant. Ces réflexions coïncident également avec les obligations de la loi GEMAPI (gestion des milieux aquatiques et protection contre les inondations) dont les collectivités territoriales vont avoir la charge au plus tard en 2018.

13. Outil financier proposé par l'Agence de l'eau de gestion contractuelle du marais sur 5 ans, venant renforcer le programme de gestion intégrée des zones humides en privilégiant 1) l'atteinte du bon potentiel des masses d'eau en 2015 (DCE) ; 2) la préservation des fonctionnalités de la zone humide (épuration des eaux, biodiversité) par le maintien du bon fonctionnement hydraulique du marais; 3) la libre circulation des espèces amphihalines (ZAP Anguille); 4) la qualité des eaux vis-à-vis du classement du marais en nouvelles zones vulnérables (Directive nitrates), de l'activité conchylicole du bassin de Marennes et des eaux de baignade de l'île d'Oléron.
Pour répondre à ces obligations et faire le lien entre le local et l'échelle de l'EPTB Charente il apparaît nécessaire de créer des sous-bassins (loi MAPTAM Loi no 2014-58 du 27 janvier 2014 de modernisation de l'action publique territoriale et d'affirmation des métropoles) gérés par l'intermédiaire d'EPAGE (établissement Public d'aménagement et de gestion de l'eau). Le marais de Brouage a été identifié comme un sous-bassin potentiel ce qui pourrait constituer une étape fondamentale dans l'organisation de la gouvernance du marais.

\section{La valorisation du marais-patrimoine par l'élevage : un nouveau modèle à inventer}

«Sauver» le marais implique également pour la quasi-totalité des acteurs le maintien de l'élevage extensif (figure 7). Et si le Conservatoire du littoral tout comme la Réserve naturelle l'ont intégré dans leur mode de gestion conventionnel des espaces naturels force est de reconnaître que cette activité est aujourd'hui peu rentable par rapport aux potentialités qu'offrent les plaines pour la céréaliculture. D'ailleurs, sur les 180 exploitations d'élevage valorisant le marais, seules $40 \%$ ont un siège d'exploitation à proximité de celui-ci et en utilisent les deux tiers de la surface, les autres se répartissant sur un rayon d'une centaine de kilomètres et disposant d'une plus grande variété de terroirs leur permettant de s'adapter. Ainsi, depuis cinq ans, de plus en plus d'exploitation se tournent vers la polyculture aux dépens de l'élevage; peu à peu le cheptel diminue et les exploitations quittent le marais. Or, le constat de la Chambre d'agriculture est qu'une fois que les vaches ont quitté le marais elles ne reviennent plus : "Les aides allonées aux éleveurs, et notamment les MAE, même les plus élevés et donc les plus contraignantes, ne permettent pas vraiment de compenser les difficultés qu'ils peuvent rencontrer au niveau de l'exploitation pour maintenir leur activité en marais, en milieu humide [...]. On force l'éleveur à rester dans un modèle d'élevage sans lui donner réellement les moyens de pouvoir palier aux contraintes» (12). Et si une évaluation des services écosystémiques est prévue dans le projet de contrat de territoire milieu aquatique afin d'identifier les services rendus par l'élevage, le mécanisme de compensation reste à inventer. 


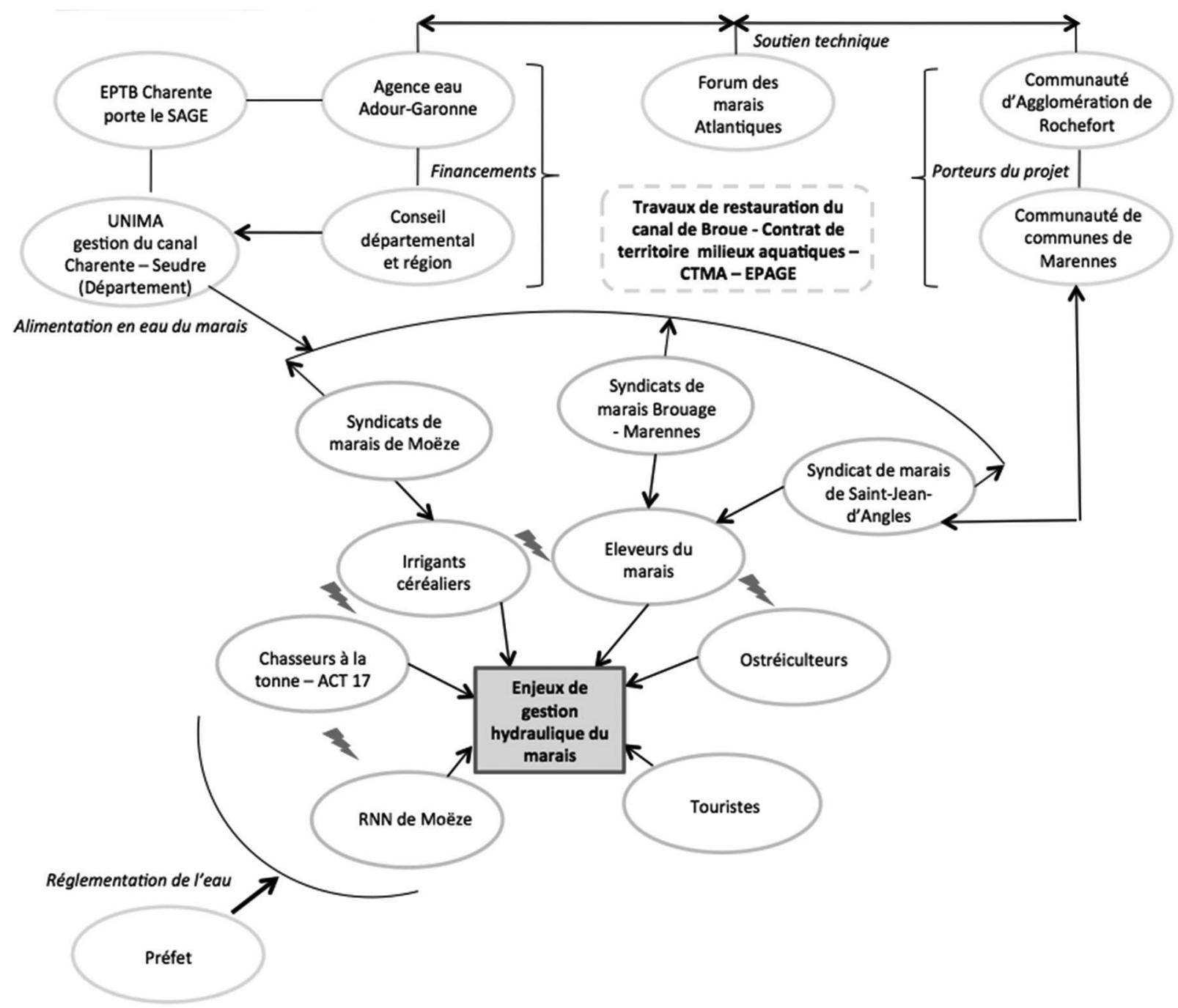

Figure 6 : Gouvernance autour des enjeux de gestion hydraulique du marais (Source : Enquête acteurs, Gouvernapat. Réalisation : Mathilde de Cacqueray - UMR ESO Angers)

NB : les figures 6, 7,8 et 9 ont été réalisées selon la méthode des cartes heuristiques à partir de l'analyse des entretiens et le repérage des enjeux récurrents (placés au centre de chaque figure) identifiés par les acteurs. Ces schémas mettent donc en relation les dires d'acteurs interrogés et leurs interactions. Ils permettent de visualiser, à un instant $t$, à partir des principaux enjeux identifiés, 1) les parties-prenantes de la gouvernance selon leurs positions, compétences, projets et actions. 2) les tensions persistantes entre acteurs dont les usages du marais diffèrent.

Governance relating to water-management issues in the marshes

De plus, les contraintes apportées par le classement de site, en sus de Natura 2000 et de la loi littoral compliquent les opportunités de diversification des activités et l'installation de nouvelles exploitations dans le marais : "La DREAL et les bâtiments de France s'opposaient complètement à un projet d'installation agricole sur Hiers. Pour eux, un site classé, ce sont des gens de l'extérieur qui viennent y mettre leurs vaches mais surtout pas de nouveaux agriculteurs qui s'installent sur la commune parce que qui dit élevage dit aussi bâtiment pour pouvoir mettre les bêtes à l'abri l'hiver. Et ça, ils sont contre [...]. À faire ce qu'ils font, à empêcher tout, le marais ne sera plus entretenu [...]. Ils veulent vraiment le mettre sous cloche le marais" (18). «Ils ont pris une photo en 2011 et dans 20 ans il faut qu'ils retrouvent la même photo. Ce qui est d'une absurdité totale parce que c'est un milieu anthropisé qui a vécu par les usages, les différents usages qui se sont succédés; il faut qu'ils comprennent à un moment ou un autre, que peut-être on changera aussi d'usages et que si on n'en change pas le marais mourra» (7). 
Figure 7 : Gouvernance autour des enjeux de valorisation de l'élevage (source : Enquête acteurs, Gouvernapat; réalisation : Mathilde de Cacqueray [UMR ESO Angers]) Governance relating to the promotion of livestock farming

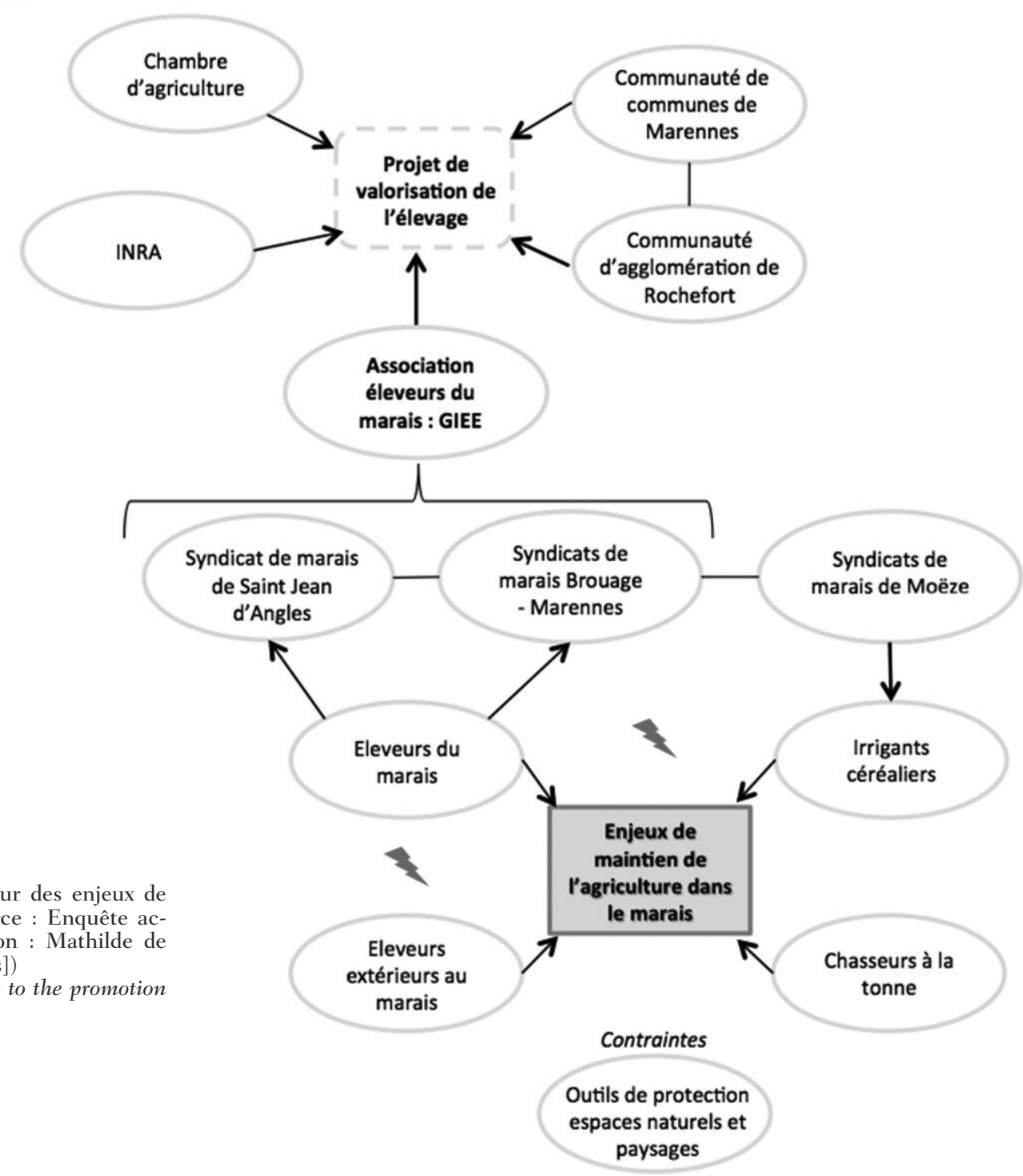

Ce nouveau régime d'habiter (Stock, 2012) issus de l'expertise écologique et paysagère, façonné au moyen de normes régissant l'organisation sociale et spatiale du marais entre donc en collision avec l'élevage, tout en contribuant paradoxalement à son maintien. Aussi, l'appel à projet déposé par la Chambre d'agriculture et appuyé par la communauté de communes de Marennes dans le cadre du CASDAR (programme national de développement agricole et rural, 2014-2017) mobilise-t-il deux associations syndicales axées sur l'élevage et une asso- ciation regroupant 34 éleveurs du marais afin de traduire cet enjeu d'adaptation organisationnelle et technique aux normes environnementales. Trois axes y sont développés dont la création d'un Groupement d'Intérêt Économique et Environnemental susceptible de financer des actions relevant de l'agro-écologie; l'organisation technique des systèmes d'élevage, en s'appuyant notamment sur le modèle des associations pastorales de montagne, et enfin la valorisation des produits de l'élevage par la labellisation et le développement des circuits courts. 


\section{Le développement touristique, levier de reconnaissance du marais et révélateur de patrimoine}

La démarche de reconnaissance et d'identification du marais de Brouage passe enfin par sa valorisation touristique : «On a ici un gros spot touristique, la citadelle de Brouage, qui draine près de 500000 visiteurs par an. Malheureusement les gens qui viennent à la citadelle ne font que transiter et ne viennent pas dans le marais. Donc la volonté qu'on a désormais c'est faire découvrir l'ensemble du marais et donc de valoriser aussi ce patrimoine différemment. On est toujours en fait sur ce triptyque qu'on avait imaginé au départ (dans le cadre du programme de la gestion intégrée des zones humides en 2008) à savoir le cour du marais: l'eau et la biodiversité qui en fait ce qu'il est, ensuite les activités économiques: l'élevage et enfin la sensibilisation et la valorisation c'est-à-dire le tourisme. Ce triptyque qu'on avait du mal à mettre en place parce qu'il fallait beaucoup d'acteurs et de l'argent, on est aujourd'hui en train de le mettre en place grâce à ce contrat territorial » (8).

Actuellement, les 3 principaux pôles touristiques que sont la citadelle de Brouage, la tour de Broue (gérées par le conseil départemental) et la réserve naturelle nationale de Moëze-Oléron (gérée par la LPO) développent leurs projets sans aucune logique territoriale vis-à-vis du marais (figure 8). Chaque acteur mène ses actions de manière isolée, partagé entre la communauté d'agglomération de Rochefort à l'initiative d'une opération Grand Site sur l'estuaire de la Charente et l'arsenal de Rochefort (pour les communes nord du marais) et la communauté de communes de Marennes (pour les communes sud) dont la compétence tourisme dépend de l'office intercommunal « île d'Oléron-Marennes ». Cette association fondée initialement sur la délimitation du bassin ostréicole de Marennes-Oléron ne masque pas les déséquilibres de notoriété, de fréquentation et d'équipement. Coincé entre les deux grands pôles touristiques du département (Oléron et presqu'île d'Arvert), en marge des grands projets d'aménagement et de développement, cette position semble d'ailleurs revendiquée par certains acteurs rencontrés (notamment les propriétaires fonciers, éleveurs comme céréaliers), traduisant une crainte du tourisme de masse mais aussi une attitude de rejet vis-à-vis de l'extérieur, susceptible de perturber l'organisation sociale et le fonctionnement interne du marais. Et s'ils s'accordent sur la nécessité d'améliorer l'accessibilité du marais pour mieux le faire découvrir, ils ne sont pas prêts non plus à l'ouvrir entièrement. Ainsi, les propos rapportés par Billaud, 1992, ne semblent pas totalement dépassés aujourd'hui « si l'agriculteur du marais de Brouage se reconnaît à une très large majorité comme le « jardinier de la nature ", il est réticent dès qu'il est question de paysage et de tourisme [...]. Ce paysage, ce « jardin » il le respecte pour son caractère patrimonial, non pas pour sa beauté, et il se méfie de sa valorisation touristique » même si les élus, notamment des deux intercommunalités apparaissent de leur côté plus sensibles aux sirènes du développement par le tourisme.

Pourtant, le seul projet structurant ${ }^{14}$ susceptible de révéler la diversité du patrimoine du marais est une piste cyclable, située le long du canal de Broue afin de relier la citadelle de Brouage à la tour de Broue où l'ancienne falaise morte du golfe de Saintonge permet de retracer la construction anthropique du marais. D'ailleurs, si la réhabilitation du chemin rural adjacent au canal récemment curé représente une réelle opportunité de diffusion des loisirs de proximité et du tourisme (promenade, observation naturaliste, itinérance à vélo via la vélodyssée), les lenteurs et la complexité administrative liées au mille-feuille de protection à prendre en compte lors du projet de réhabilitation hydraulique du canal laissent entrevoir de nouvelles difficultés pour la collectivité « pour le canal de Broue, j’ai mis pratiquement 16 mois d'instruction avant d'avoir les autorisations pour pouvoir lancer le marché et commencer les travaux... C'est un site classé donc ça passe jusqu'au bureau du premier ministre. Notre dossier d'étude réglementaire faisait 350 pages. Parce qu'il fallait une étude d'impact, une étude d'incidence loi sur l'eau, une évaluation d'incidences Natura 2000 et donc une autorisation site classée. On arrive à des dossiers énormes, qui sont indigestes et avec des délais réglementaires qui à chaque fois s'ajoutent en plus. Et ça, les acteurs $d u$ territoire ils ne comprennent pas, pour eux c'est absurde» (8).

14. Projet ayant évolué depuis nos enquêtes sous l'initiative de la communauté d'agglomération de Rochefort et de la communauté de Marennes autour d'un « Grand-projet marais de Brouage » axé «sur la préservation de la richesse exceptionnelle des lieux mais également le développement des potentiels touristiques et économiques « perennisateurs » de la qualité exceptionnelle de cette zone remarquable » (document de travail, non paginé) 
Figure 8 : Gouvernance autour des enjeux de valorisation touristique du marais (source : Enquête acteurs, Gouvernapat. Réalisation : Mathilde de Cacqueray [UMR ESO Angers]) Governance relating to the promotion of tourism in the marshes

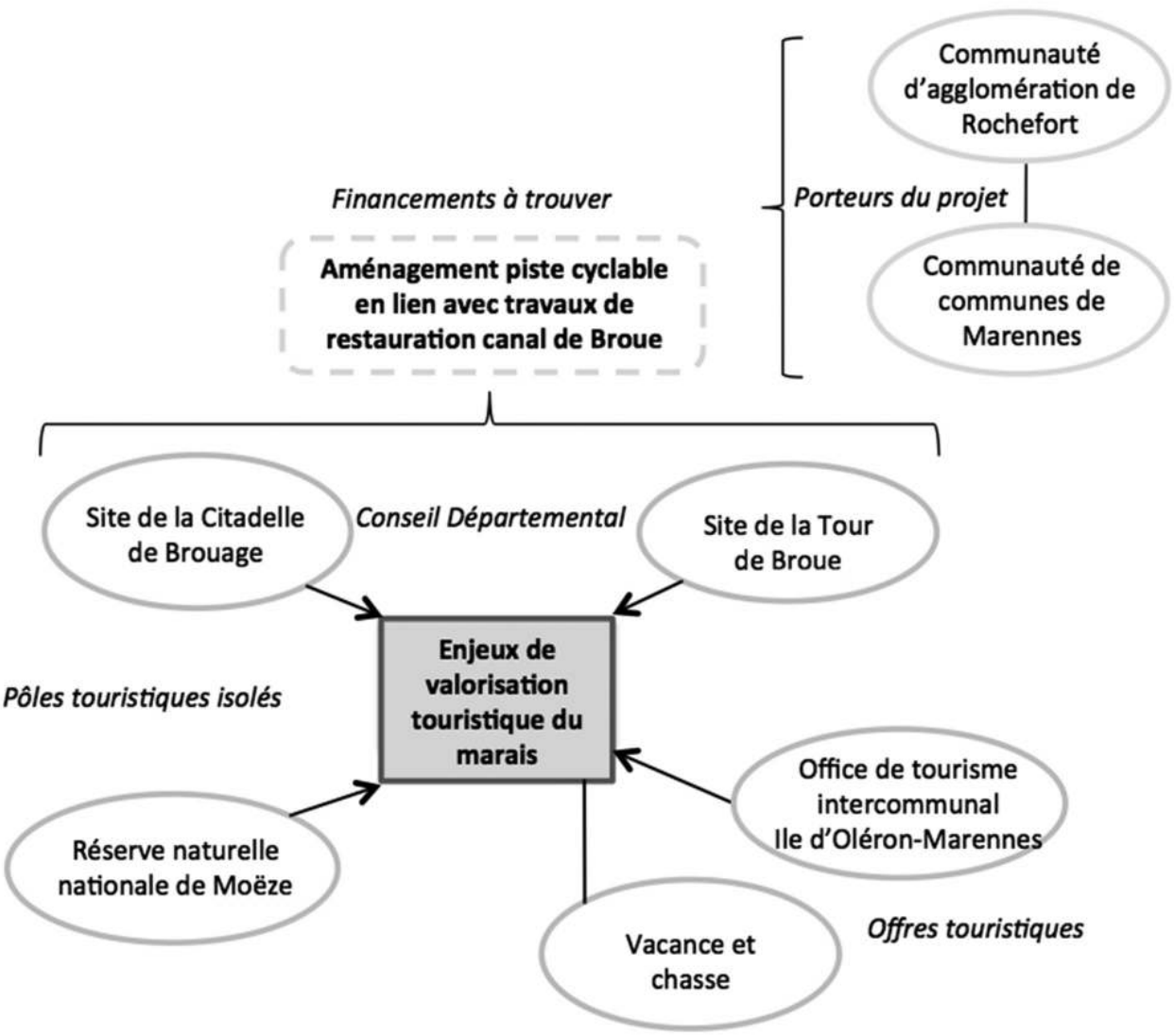

\section{La conservation de la biodiversité : vers une gestion intégrée?}

Focalisés sur la création de la réserve nationale dans les années 1980, élargis à l'ensemble du marais grâce au périmètre de préemption et aux acquisitions foncières du Conservatoire du littoral dans les années 1990, les enjeux de conservation de la biodiversité se sont ensuite diffusés de manière réticulaire à l'ensemble des zones humides et côtières du littoral sous l'impulsion de Natura 2000. Vécue localement comme une véritable "effraction exogène ", l'application des directives habitats et oiseaux dans le marais de Brouage (désignation des ZPS et ZSC en 2004 et 2009) peut néanmoins être considérée comme le premier acte fondateur d'une gouvernance de la biodiversité dans le marais. Ainsi, le choix de la communauté de communes de Marennes d'en être l'opérateur à partir de 2010 a permis à la collectivité de se saisir de cet enjeu (et d'en neutraliser les conflits) afin de jouer un rôle d'interface entre la DREAL, les acteurs socio-économiques du marais et les acteurs de l'environnement pour la réalisation du document d'objectifs et sa mise en œuvre (Communauté de communes de Marennes, 2012). D'ailleurs, dès 2008, cette même collectivité s'engageait dans un programme de gestion intégrée des zones humides permettant d'associer enjeux écologiques et gouvernance locale à travers un programme d'actions territorialisés ciblées sur la gestion de la ressource, la préservation de la biodiversité et la valorisation économique et culturelle (Pouzin, 2011). 
Cependant, force est de reconnaître que cet enjeu de conservation de la biodiversité divise et reste inégalement approprié par les acteurs interviewés, pour qui la valeur écologique du marais reste fondamentalement attachée à des usages et à l'histoire du marais (cf. partie 1). De plus, tous les acteurs de la protection de l'environnement (figure 9) n'ont pas la même manière de faire en matière de conservation et si les approches peuvent être complémentaires, des rivalités existent pour garder « sa » place et «sa » légitimité dans le paysage de la conservation. L'arrivée d'un nouvel acteur, l'Agence des aires marines protégées et le Parc naturel marin est ainsi perçu comme une opportunité par certains afin de rendre plus cohérente la gestion de l'interface terre mer ${ }^{15}$, mais menaçante pour d'autres : "Les réserves seront dans le Parc... donc comment nos outils seront utilisés, manipulés pour en faire des réserves qui ne seront que sur le papier? C'est un peu l'inquiétude, que les réserves gardent leur entité au sein d'un parc qui ne va pas avoir d'orientation très forte en termes de protection du patrimoine parce ce sera toujours dans la discussion. Un moment donné voilà, en réserve naturelle on arrive encore un peu à avoir, voilà, des choses comme des réserves intégrales avec du personnel et on arrive à faire en sorte que ce soit respecté. Et on sent que le Parc marin ça peut vite déraper quoi. Donc on est un petit peu inquiet» (13). La concertation autour des enjeux de conservation de la biodiversité semble donc différemment appréciée et ce, d'autant plus, qu'elle s'est engagée dans un contexte particulier où la sélection des marais de Brouage par le ministère de l'Environnement, de l'Energie et de la Mer en 2011 pour la création d'un Parc national de zone humide ${ }^{16}$, sans communication ni consultation locale, pendant la phase de concertation sur le Parc national marin, l'écriture du Docob Natura 2000, et le classement du site a marqué la mémoire collective : "On était bien partie jusqu'en 2011 sur un projet de création d'un syndicat mixte de gestion intégrée du marais de Brouage; ça plante une première fois, normal ces choses-là, ça infuse. Mais ce qui est

15. Cf. figure 3 où l'on observe une rupture dans les représentations des périmètres patrimoniaux entre les parties amont et aval du marais et plus globalement entre le marais et son aire marine adjacente.

16. Prévue dans le plan national en faveur des zones humides de 2010 (MEEDDN, 2010) afin d'accélérer la préservation des zones humides les plus sensibles. Abandonnée - sans autre précision - lors de l'évaluation du Plan national d'action pour les zones humides 2010-2013 (Rapport $\mathrm{n}^{\circ}$ 008343-01 Évaluation du Plan national d'action pour les zones humides 2010-2013 PNZH). très dommage c'est qu'on avait autour de la table la LPO, les chasseurs, l'État, la région, machin... tout le monde! Et puis on apprend par un communiqué de presse que le ministère de l'Environnement nous verrait bien lauréat d'un appel à projet pour un Parc national de zones humides et là vous mettez 300 types dans les salles des fêtes, c'est fini [...]. L'État a, mon avis, un rôle fondamental à jouer sur la préservation des espaces les plus sensibles mais pas comme ça, pas comme ça, pas en cassant les dynamiques locales » (6).

Les injonctions multiples de la part de l'état et de ses services et leur intervention dans le milieu local ont ainsi rendu les acteurs frileux voire réfractaires à tous projets supplémentaires de protection, considérant certaine approche de conservation comme contre-nature par rapport à la construction anthropique du marais : "Je me suis fâché parce qu'on dit: Natura 2000, il faut revenir à l'état initial. Je dis : qu'est-ce que vous appelez l'état initial? Parce moi je veux bien qu'on revienne à l'état initial mais faut dire lequel. Parce que si on revient à l'état initial ici ça va être vite réglé... il y a 1000 ans c'était un golfe, il y avait de l'eau de mer. Donc on revient à l'état initial? Non mais attendez... (17). Et si la question du partage entre eaux douce et salée ne se pose pas en amont du marais, elle est d'actualité dans la partie aval, notamment au niveau de la Réserve nationale de Moëze dont la digue à la mer est régulièrement rompue par les tempêtes : "Oui, ça va changer... et le Conservatoire n'est pas un propriétaire comme un autre. Le terrain, s'il repart à la mer, on transforme un écosystème par un autre écosystème. Et parfois le nouvel écosystème il est plus riche que le précédent. Donc [d'un point de vue écologique] ça ne nous pose pas de problème particulier. Pour reprendre l'exemple de la réserve, une partie va passer à la mer, et même au sein de l'organisme gestionnaire, les positions sont très diverses. Il y a des gens qui veulent conserver la digue, d'autre pas. Donc ce qui prouve que d'un strict point de vue écologique les façons de voir sont différentes. Je ne vous parle pas de l'agriculteur qui est derrière et qui va voir son champ de maïs recouvert par l'eau salée. Lui, il sait ce qu'il veut faire... Après, il y a la position des communes, il y a la position de l'État, il y a la position de plein de gens, du Conservatoire. C'est très compliqué » (2). La conservation en l'état de cette partie du marais fait donc l'objet de questionnements face au changement climatique et à ses répercussions, changement qui, rappelons-le, ne 


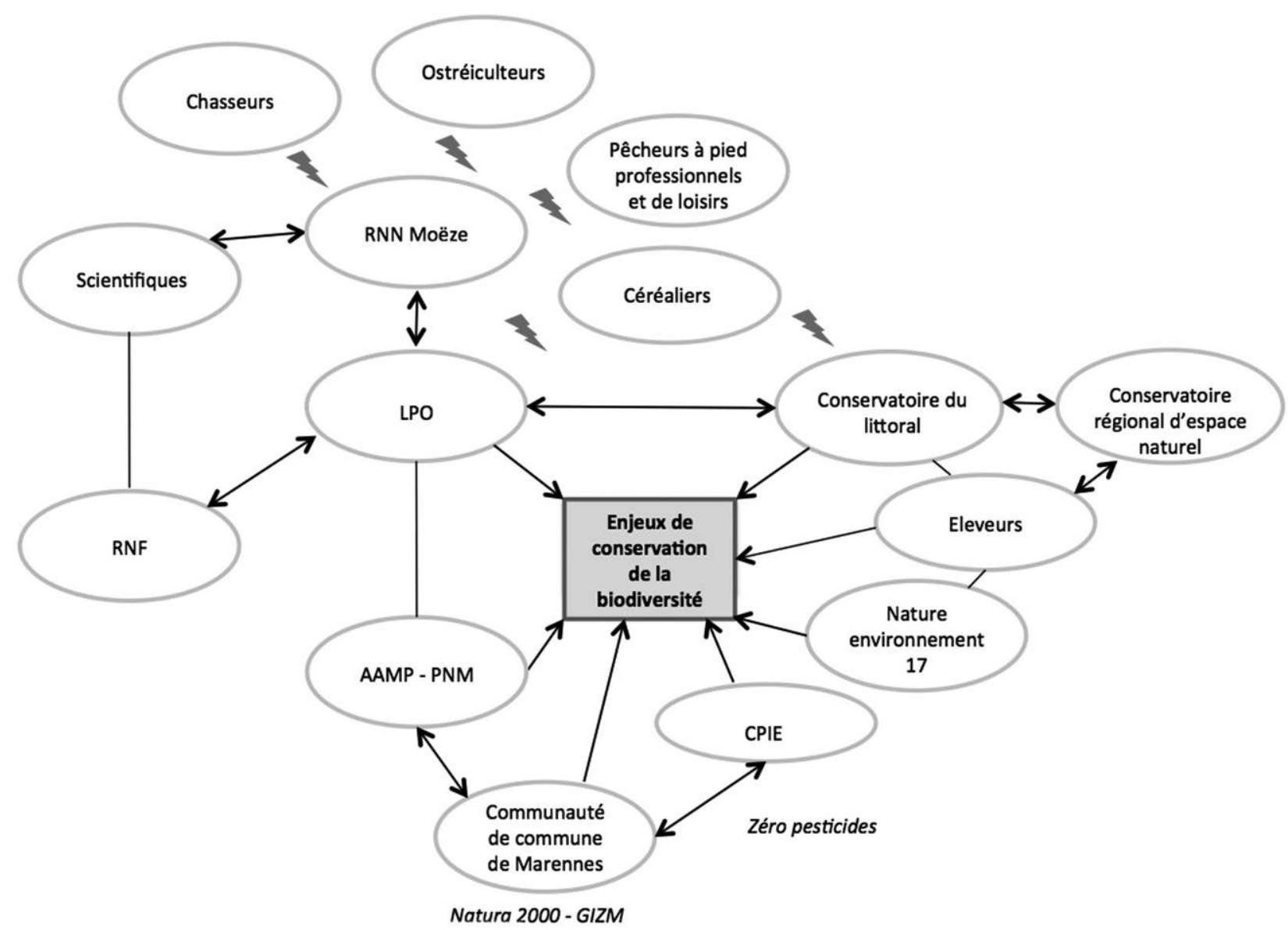

Figure 9 : Gouvernance autour des enjeux de conservation de la biodiversité du marais (Source : Enquête acteurs, Gouvernapat; Réalisation Mathilde de Cacqueray - UMR ESO Angers)

Governance relating to biodiversity conservation in the marshes

semblent pas perçu comme une menace pour les usagers du marais puisque seuls les services de l'état et les élus l'ont abordé dans nos entretiens.

\section{Conclusion}

L'analyse de ce qui fait patrimoine pour les différents acteurs du marais permet de mesurer les décalages de perception et d'appréciation des valeurs accordées à la nature entre, d'une part, les tenants d'une approche écocentrée (Hatem, 1990; Sébastien, 2004) privilégiant la conservation des écosystèmes stricto sensu et, d'autre part, les catégories développant une approche anthropocentrée insistant sur leur caractère culturel et la valorisation des ressources et services associés. Elle fait également ressortir des conceptions différenciées du patrimoine à transmettre, entre idéalisation de la stabilité (tant écologique que paysagère) au nom d'un héritage souvent perçu comme figé, et affirmation d'un patrimoine vivant, produit d'une histoire sociale, naturelle et humaine, en mouvement.

Pourtant, au-delà de ces dualités à l'origine des principaux conflits, des convergences apparaissent autour d'une vision partagée du marais vu comme un «tout » indissociable, un « ensemble » aux usages et fonctionnalités multiples et interdépendants à sauvegarder. C'est d'ailleurs face à la menace de disparition des structures hydrauliques et de l'organisation sociale de cet ensemble que de nouvelles proximités et ententes se créent entre acteurs, autour d'enjeux communs localisés, dans un contexte global où le modèle de la zone humide en redéfinit les contours et les finalités. Ainsi, la gouvernance territoriale qui se met progressivement en place autour de la gestion intégrée des zones humides peut être lue comme une forme d'appropriation et d'ajustement local à/ de ces enjeux, tout en légitimant le rôle et la place 
de certaines catégories jusque-là peu valorisées dans les processus décisionnels, tels les éleveurs (et leurs syndicats marais), les acteurs de la protection de nature et même des touristes sur lesquels les territoires misent désormais.

Elle pose néanmoins la question des modalités d'adaptations technique, organisationnelle et institutionnelle de ce projet dans un monde rural où l'apprentissage de la concertation/négociation et la mobilisation des habitants autour des questions relatives à l'environnement et à la nature restent peu développés. Car si les espaces protégés et la biodiversité participent désormais du maillage de l'aménagement du territoire et doivent y contribuer (Laslaz, 2012; Franchomme et al., 2013), leur acceptation sociale nécessite de nouvelles formes de médiation et de gouvernance. En ce sens, l'approche patrimoniale, par définition transversale et parce qu'elle permet de décloisonner les savoirs et les représentations peut constituer un outil pertinent de médiation territoriale.

\section{Remerciements}

Ce travail a été financé par le programme «Quels littoraux pour demain? » 2013-2017 de la Fondation de France dans le cadre du projet « Gouvernance et développement durable des espaces du patrimoine naturel littoral »[https://gouvernapat.hypotheses. org/].

\section{Bibliographie}

Barthon C., Chadenas C., de Lajartre A., Pancher A., 2013. Valeur, fonction et médiation dans les espaces naturels sensibles : une patrimonialisation inachevée de la nature. Exemples dans les Pays de la Loire, VertigO - la revue électronique en sciences de l'environnement [En ligne], Horssérie 16, juin 2013, mis en ligne le 30 mai 2013, consulté le 11 novembre 2017.

Berdoulay V., Soubeyran O., 2013. Sens et rôle du patrimoine naturel à l'heure de l'aménagement durable et du changement climatique, L'Espace géographique, 4/2013 (Tome 42), p. 370-380.

Billaud J.-P., Bruguière-Garde Y., 1992. L'homme, l'eau, le marais : quelques aspects d'une relation sociale dans une zone humide, Économie rurale, no 208-209, p. 50-53.
Billaud J.-P., 1996. Négociations autour d'une nature muette. Dispositifs environnementaux dans les marais de l'Ouest. Études rurales, no 141-142, p. 63-83.

Bossuet L., Boutry O., 2012. Ressource en eau. Illustration à partir du littoral charentais, Économie rurale Agricultures, alimentations, territoires, $\mathrm{n}^{\circ} 332$, p. 74-87.

Bouba-Olga O., Boutry O., Rivaud A., 2009. Un approfondissement du modèle exit-voice par l'économie de la proximité, Natures Sciences Sociétés, 4/2009 (vol. 17), p. 381-390.

Bouisset C., Degrémont I., 2013-a. La patrimonialisation de la nature : un processus en renouvellement, L'Espace géographique, 2013/3, tome 42, p. 193-199.

Bouisset C., Degrémont I., 2013-b. Patrimonialiser la nature : le regard des sciences humaines, VertigO - la revue électronique en sciences de l'environnement [En ligne], Hors-série 16 | juin 2013, mis en ligne le 16 avril 2013, consulté le 2 février 2016 [http://vertigo.revues.org/13542].

Bouisset C., Degrémont I., 2013-c. Construire un patrimoine naturel : valeurs (de société) contre critères (officiels)? L'exemple de hauts lieux montagnards pyrénéens, VertigO - la revue électronique en sciences de l'environnement [En ligne], Hors-série 16, juin 2013, mis en ligne le 30 mai 2013, consulté le 6 novembre 2017 [http://vertigo.revues. org/13750].

Bry C., Hoflack P., 2004. Le bassin versant de la Charente : une illustration des problèmes posés par la gestion quantitative de l'eau, Courrier de l'environnement de l'INRA, n ${ }^{\circ} 52$, septembre, p. 81-96.

Cadoret A., 2011. Analyse des processus conflictuels. Le cas du Languedoc-Roussillon, L'Espace géographique, 3, tome 40, p. 231-244.

Candau J., Deldrève V., Deuffic P., 2012. Publicisation contrôlée de problèmes territoriaux autour de l'eau. SociologieS [Online], Theory and research, Online since 27 January 2012, connection on 23 November 2016 [http://sociologies. revues.org/3822].

Claeys-Mekdade C., 2000, Les conflits d'aménagement. Rapports à la «nature » et rapports sociaux. La Camargue, un cas révélateur. Thèse de doctorat en sociologie, Université de Provence, Aix-en-Provence, 405 p.

Claeys C., 2014. Créer un parc national des Calanques : préserver, partager ou confisquer un patrimoine socio-naturel? Annales de Géographie, nº 698, vol. 4, p. 995-1015.

Corlay J.-P., 1996. Les marais maritimes de la Vilaine à la Gironde : géosystème maraîchin atlantique et promesses aquacoles, Norois, vol. 132, n 1, p. 547-569.

De Lajartre A., Barthon C., Andreu-Boussut V., Chadenas C., Michel X., 2016. Habiter les habitats (naturels) : quelle place pour l'homme au sein du patrimoine naturel littoral?, in Robert S., Melin H. (dir.), Habiter le littoral Enjeux contemporains, Presses universitaires de Provence, coll. «Espace et développement durable », p. 39-55.

Depraz S., 2008. Géographie des espaces naturels protégés, Paris, Armand Colin, coll. « U géographie », 320 p.

Depraz S., 2015. Dix méthodes pour mesurer l'acceptation sociale autour des espaces naturels protégés, in LASLAZ L., GAUCHON C., Duval M., Héritier S. (dir.), Espaces protégés et terri- 
toires, Conflits et acceptation, Paris, Belin, coll. « Mappemonde », p. 49-66.

EPTB Charente (Institution interdépartementale pour l'aménagement du fleuve Charente et de ses AFFluents), 2014. Schéma d'aménagement et de gestion des eaux de la Charente, diagnostic, 109 p.

Franchomme M., Bonnin M., Hinnewinkel C., 2013. La biodiversité « aménage-t-elle » les territoires? Vers une écologisation des territoires, Revue Développement durable et territoires, Vol. 4, n 1, avril 2013, consulté le 19 novembre 2014 [http://developpementdurable.revues.org/9749].

Gravarri-Barbas M. (dir.), 2005. Habiter le patrimoine : enjeux, approches, vécu, Rennes, PUR, 618 p.

Gilardeau J.-M., 2008. La gestion des zones humides par les Associations Syndicales de Propriétaires: Guide de mise en conformité des statuts des Associations Syndicales de Propriétaires en zones Humides, Forum des Marais Atlantiques, $133 \mathrm{p}$.

Натем F., 1990. Le concept de développement soutenable. Économie prospective internationale, $\mathrm{n}^{\circ} 44$, p. 101-117.

Héritier S., Guichard-Anguis S., 2008, Le patrimoine naturel, entre culture et ressource, présentation, Géographie et Cultures, Paris, L'Harmattan, vol. 66, p. 3-10.

Kalaora B., 2010. Les zones humides et le Conservatoire du littoral : perceptions et cadre d'expérience, L'Espace géographique, 4/2010, tome 39, p. 361-374.

Laslaz L., Gauchon C., Duval M., Héritier S. (dir.), 2015. Les espaces protégés. Entre conflits et acceptation, Paris, Belin, $432 \mathrm{p}$.

Laslaz L. (dir.), 2012. Atlas mondial des espaces protégés : la société face à la nature, Paris, Autrement, $96 \mathrm{p}$.

Lepart J., Marty P., 2006. Des réserves de nature aux territoires de la biodiversité, Annales de Géographie, tome 115, $n^{0}$ 651, 2006, p. 485-507.

Maris V., Roche Ph., Levrel H., Geijzendorffer L., 2016. Regards croisés sur les valeurs de la biodiversité et les services écosystémiques : les valeurs en question, in Roche Ph. et al. Valeurs de la biodiversité et les services écosystémiques, perspectives interdisciplinaires, Versailles, Edition Quae, p. 21-38.

Ministère de l'Écologie, de L'Énergie, du DÉveloppement DURABlE ET DE LA MER, 2006. Les zones humides: un enjeu national. Bilan de 15 ans de politiques publiques, $95 \mathrm{p}$.

Ministère de l'Écologie, de L'Énergie, du Développement durable et de La Mer, 2010. Plan national d'action en faveur des zones humides, février 2010, 28 p.

Ollier C., Provost S., 2009. Projet de classement de l'ancien golfe de Saintonge, DREAL Poitou-Charentes, $74 \mathrm{p}$.

PÉRON F. (dir.), 2002, Le patrimoine maritime, construire, transmettre, utiliser, symboliser les héritages maritimes européens, Rennes, PUR, 538 p.
Pouzin L., 2011. Programme d'Actions Territorialisées des marais de Brouage et de l'Estuaire de la Seudre, Communauté de Communes du Bassin de Marennes, Service «Zones Humides ». $239 \mathrm{p}+$ Fiches-action.

Réault-Mille S., 2003. Les marais charentais. Géohistoire des paysages du sel, Rennes, PUR, 2003, 270 p.

Rey-VALEtTe H. et al., 2014. Comment analyser la gouvernance territoriale? Mise à l'épreuve d'une grille de lecture, Géographie, économie, société (n $\mathrm{n}^{\circ}$ 16), Lavoisier, p. 65-89.

Rivaud A., 2009. Conflits d'usage autour de la ressource en eau : quelles stratégies pour les ostréiculteurs du bassin de Marennes-Oléron, Colloque international «Les $6^{e}$ journées de la proximité : le temps des débats », octobre 2009, Poitiers, France [https://hal.archives-ouvertes.fr/hal-00547207].

Robert S, Melin H. (dir.), 2016. Habiter le littoral : enjeux contemporains, Presses Universitaires de Provence, Géographie de l'environnement, coll. « Espace et développement durable », $476 \mathrm{p}$.

Sébastien L., Brodhag C., 2004. À la recherche de la dimension sociale du développement durable. Développement durable et territoires [En ligne], Dossier $n^{\circ} 3$, consulté le 29 septembre 2016 [http://developpementdurable.revues. org/1133].

SÉbastien L., 2016. L'attachement au lieu, vecteur de mobilisation collective? Étude de cinq territoires ruraux, Norois, PUR, no 238-239, p. 23-41

Sтоск M., 2012, Faire avec de l'espace : pour une approche de l'habiter par les pratiques. in Frelat-Kahn B., Lazzarotti O. (dir.), Habiter Vers un nowveau concept, Paris, Armand Colin, p. 57-74.

Torre A., Melot R., Bossuet L., Cadoret A., Caron A., Darly S., Jeanneaux Ph., Thierry Kirat, Vu Pham H., 2010. Comment évaluer et mesurer la conflictualité liée aux usages de l'espace? Éléments de méthode et de repérage, VertigO - la revue électronique en sciences de l'environnement [En ligne], Volume 10, Numéro 1, avril, mis en ligne le 30 avril 2010, consulté le 15 septembre 2017 [http://vertigo. revues.org/9590].

Veschambre V., Ripoll F., 2005. L'appropriation de l'espace comme problématique, Norois, no 196-2, p. 7-15.

Veschambre V., 2007. Le patrimoine : un objet révélateur des évolutions de la géographie et de sa place dans les sciences sociales, Les Annales de géographie, nº 656, p. 361-381.

Veschambre V., 2008, Traces et mémoires urbaines, enjeux sociaux de la patrimonialisation et de la démolition, Rennes, PUR, 315 p. 
Planche I (Mathilde DE CACQUERAY et al. - Le patrimoine, un élement fédérateur pour la gouvernance des espaces protégés?)
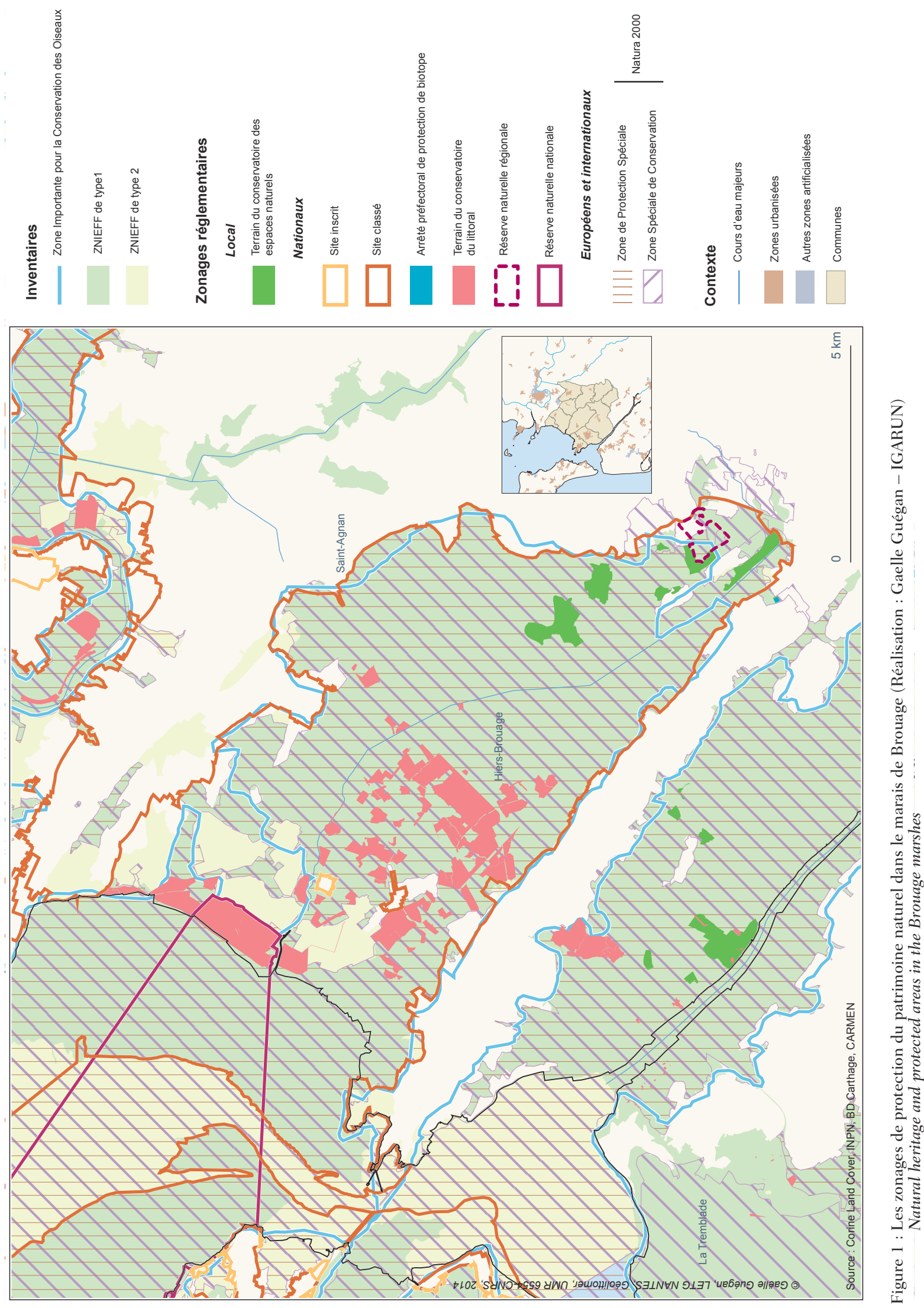


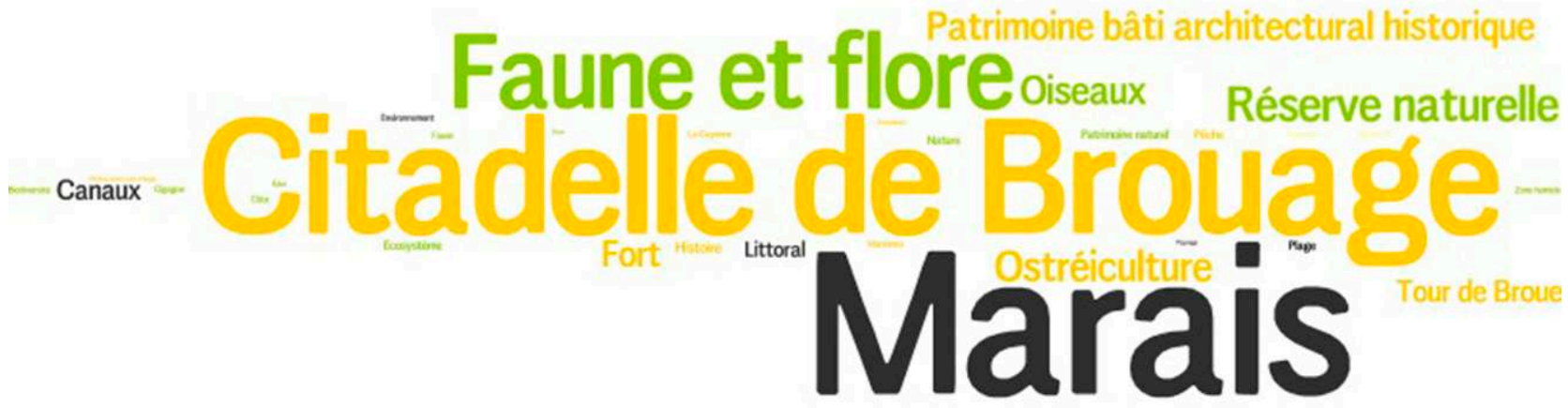

Figure 3 : Mots associés au patrimoine par les habitants fréquentant le marais (Source : Enquête habitants, Gouvernapat. Total 200 questionnaires, 362 mots cités, 312 traités. Conception Céline Barthon)

Légende : en noir, les mots se référant au milieu géographique; en vert à la nature et ses infrastructures (Réserve naturelle nationale); en orange au patrimoine culturel, historique et technique. Parmi les 362 mots cités, seuls ceux nommés au moins 3 fois ont été traités (312). La taille des mots est proportionnelle au nombre de citations.

Words associated with heritage by inhabitants who use the marshes

Figure 4 : Délimitation spatiale du patrimoine du marais de Brouage par les acteurs (Source : enquête acteurs, Gouvernapat; Réalisation : Simon Charrier IGARUN)

Lecture : la carte représente la superposition des différents périmètres dessinés par les acteurs interrogés.

$$
\text { Actors' spatial delineation of }
$$

heritage areas in the Brouge marshes

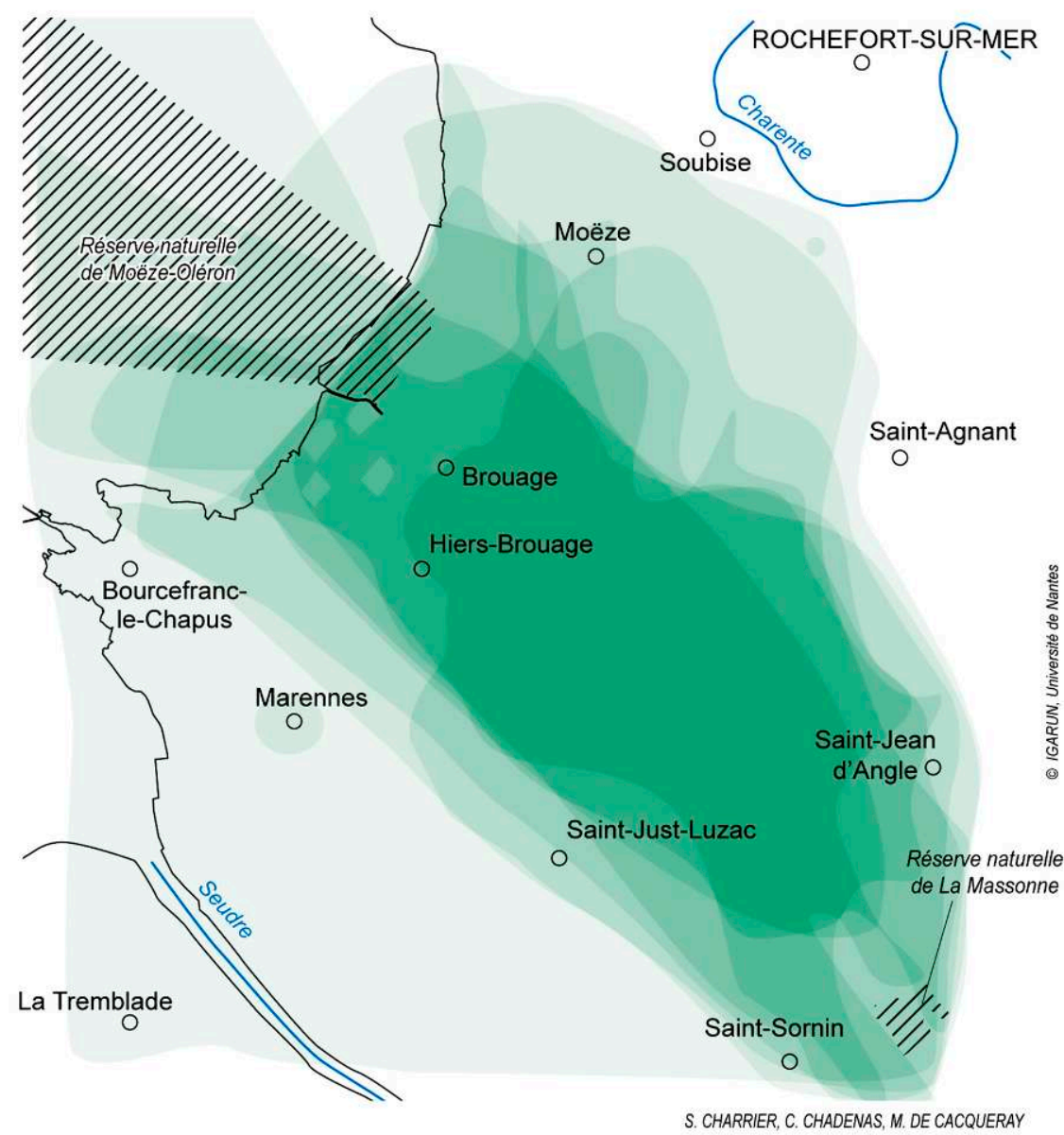

\section{Nombre de citations}

$\begin{array}{llllllll}1 & 2 & 3 & 4 & 5 & 6 & 7 & 8\end{array}$

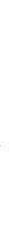

